\title{
The Minimal Robust Positively Invariant Set for Linear Difference Inclusions and its Robust Positively Invariant Approximations
}

\author{
S. V. Raković, K. I. Kouramas, E. C. Kerrigan, J. C. Allwright and D. Q. Mayne* \\ Technical Report EEE/C\&P/SVR/9-d/2005, Imperial College London, 2005
}

\begin{abstract}
Robust positively invariant (RPI) sets for linear difference inclusions are considered here under the assumption that the linear difference inclusion is absolutely asymptotically stable in the absence of additive state disturbances, which is the case for parametrically uncertain or switching linear discrete-time systems controlled by a stabilizing linear state feedback controller. The existence and uniqueness of the minimal RPI set and the minimal convex RPI set are studied. A new method for the computation of outer RPI approximations of the minimal RPI set for linear difference is presented; these approximations include a family of star-shaped RPI sets and two families of convex RPI sets. The use of a family of star-shaped RPI sets, and the characterization of the family, is reported for the first time.
\end{abstract}

Keyword: Set invariance, Minimal Robust Positively Invariant Set, Linear Difference Inclusions

\section{Introduction}

One of the fundamental tools employed in robust control of constrained dynamical systems is set invariance theory [1]. This is used in the design of reference governors [2] and predictive controllers [3-5] to guarantee constraint satisfaction, stability and convergence properties. One technique for robust control of constrained discrete-time systems is robust time-optimal control [6-9], which is based on the computation of a sequence of robust control (positively) invariant (RCPI) sets when the target set is also a RCPI set. A suitable target set in robust time-optimal control is the minimal robust positively invariant (mRPI) set [10]. The relevance of these ideas is demonstrated in the novel robust predictive controllers proposed recently in [11-13].

Computational issues and algorithmic procedures for the calculation of the (RCPI) sets and the application of these in robust control for constrained systems are discussed by a number of researchers $[10,14-22]$. One of the outstanding problems for autonomous linear discrete-time systems is exact characterization of the mRPI set $[1$, 10,23]. Several authors have developed procedures for the computation of outer approximations, with prespecified accuracy, of the mRPI set which are themselves RPI sets ; see, for instance, a procedure proposed in [24] and an alternative, simpler and improved, procedure in [25]. However, these papers address only approximation techniques for autonomous linear discrete-time invariant systems.

Linear difference inclusions (LDI) are used for modeling linear systems with parametric uncertainty; see, for example, [26,27], and linear systems with switching dynamics [28].

\footnotetext{
*This research was supported by the Engineering and Physical Sciences Research Council and the Royal Academy of Engineering, United Kingdom.

${ }^{\dagger}$ S. V. Raković, K. I. Kouramas, J. C. Allwright and D. Q. Mayne are with Imperial College London, London SW7 2BT, United Kingdom, email: \{k.kouramas, sasa.rakovic, j.allwright, d.mayne\}@imperial.ac.uk.

${ }^{\ddagger}$ E. C. Kerrigan is a Royal Academy of Engineering Research Fellow and is with the Department of Engineering, University of Cambridge, CB2 1PZ Cambridge, United Kingdom, email: erickerrigan@ieee.org.
} 
Uncertainty in the state transition matrix raises several issues, e.g. non-convexity of the forward reachable sets (as suggested by results reported in [26]). Such phenomena do not occur in the simpler case of autonomous linear discrete-time systems subject to bounded additive state uncertainty.

We establish that (i) under mild assumptions, the exact forward reachable sets are star-shaped sets [29,30] , (ii) existence and uniqueness of the mRPI set and (iii) existence of three families of outer RPI approximations of the mRPI set. Non-convexity and computational complexity issues regarding the exact mRPI set are addressed by establishing existence and uniqueness of the minimal convex robust positively invariant (mCRPI) set. Additionally, we discuss computation of outer RPI approximations of the mCRPI set. Some technical and notational errors that appear in the conference version [31] of this work are corrected in this technical report. This technical report is based on the journal version of the paper [32].

Since the exact mRPI set is generally non-convex and it is very difficult to obtain a simple computational scheme for constructing it, three families of RPI sets are introduced. One consists of star-shaped RPI sets. This seems to be the first time that star-shaped RPI sets have been defined and studied. The other families consist of convex RPI sets: one contains convex sets that are outer-bounds for the mRPI set and the other contains the set that is tightest convex approximation of the MCRPI set as well as other convex sets.

\section{Paper Structure}

Section 2 is concerned with preliminaries. Existence and uniquness of the mRPI and the mCRPI sets are established in Section 3 . Section 4 contains a characterization of three families of RPI sets, a study of the limiting behaviour of increasingly accurate RPI approximations and a condition for characterization of a family of outer RPI approximations of the mCRPI set. Computational procedures for the case when the disturbance set is a polytope are given in Section 5 and some illustrative examples are in Section 6. Section 7 contains some conclusions. All proofs are given in the Appendix.

\section{Basic Nomenclature and Definitions}

Let $\mathbb{N} \triangleq\{0,1,2, \ldots\}, \mathbb{N}^{+} \triangleq\{1,2, \ldots\}$ and $\mathbb{N}_{q} \triangleq\{0, \ldots, q\}, \mathbb{N}_{q}^{+} \triangleq\{1, \ldots, q\}$ for $q \in \mathbb{N}^{+}$. Further $\mathbb{B}_{p}^{n}(\gamma) \triangleq\{x \in$ $\left.\mathbb{R}^{n} \mid\|x\|_{p} \leq \gamma\right\}$, where $\|\cdot\|_{p}$ denotes the vector $p$-norm, and $\mathbb{R}_{+}^{n} \triangleq\left\{x \in \mathbb{R}^{n} \mid x \geq 0\right\}$. Given an integer $s \in \mathbb{N}^{+}$and sets $\Omega_{i} \subset \mathbb{R}^{n}$, the Minkowski set addition is defined by $\bigoplus_{i=1}^{s} \Omega_{i} \triangleq \Omega_{1} \oplus \Omega_{2} \oplus \ldots \oplus \Omega_{s}=\left\{\sum_{i=1}^{s} \omega_{i} \mid \omega_{i} \in \Omega_{i}\right\}$. For $\Omega \subset \mathbb{R}^{n}$, interior $(\Omega)$ and $\operatorname{co}(\Omega)$ denote the interior and convex hull of $\Omega$ and $\lambda \Omega \triangleq\{\lambda \omega \mid \omega \in \Omega\}$ for any $\lambda \in \mathbb{R}$.

To clarify our use of the polyhedral/polytopic, $C$ and star-shaped sets we recall the following definitions:

Definition 1 (Polyhedron/Polytope) A polyhedron is the intersection of a finite number of open and/or closed half-spaces. A polytope is a closed and bounded polyhedron.

Definition 2 ( $C$ set) $A$ set $\Omega \subset \mathbb{R}^{n}$ is a $C$ set if it is a compact, convex set that contains the origin in its interior.

Definition 3 (Star-Shaped set) $A$ set $\Omega \subset \mathbb{R}^{n}$ is a star-shaped set with a center at $\omega_{c} \in \mathbb{R}^{n}$ if $\omega_{c} \in \Omega$ and $\left\{\omega_{c}\right\} \oplus \lambda\left(\left\{-\omega_{c}\right\} \oplus \Omega\right) \subseteq \Omega$ for all $\lambda \in(0,1]$. A star shaped set $\Omega \subset \mathbb{R}^{n}$ is basic if $\omega_{c}=0$.

We say that a set calculation is practicable when it can be carried out in finite time.

\section{Preliminaries}

We consider throughout the following linear difference inclusion:

$$
\begin{aligned}
x^{+} & \in \mathcal{D}(x, \mathbb{A}, \mathbb{W}) \\
\mathcal{D}(x, \mathbb{A}, \mathbb{W}) & \triangleq\{A x+w \mid A \in \operatorname{co}(\mathbb{A}), w \in \mathbb{W}\} \\
\mathbb{A} & \triangleq\left\{A_{i} \in \mathbb{R}^{n \times n} \mid i \in \mathbb{N}_{q}^{+}\right\}
\end{aligned}
$$


where $x \in \mathbb{R}^{n}$ is the current state, $x^{+}$is the successor state, $w \in \mathbb{R}^{n}$ is an unknown disturbance and $q \in \mathbb{N}^{+}$is a finite integer. The system is subject to an external additive state disturbance $w$ that is contained in a $C$-set $\mathbb{W} \subset \mathbb{R}^{n}$. The system transition matrix $A$ is uncertain and is known only to the extent that, at each time, it belongs to the convex hull of a finite set $\mathbb{A}$ of known and bounded matrices $A_{i}$ so

$$
A=\sum_{i=1}^{q} \lambda_{i} A_{i}, \quad \lambda \in \Lambda
$$

where $\lambda$ can vary with time and

$$
\Lambda \triangleq\left\{\lambda \in \mathbb{R}_{+}^{q} \mid \sum_{i=1}^{q} \lambda_{i} \leq 1\right\}
$$

We adopt the following standing assumption:

Assumption 1 (i) The set $\mathbb{W}$ is a $C$-set in $\mathbb{R}^{n}$ and

(ii) The matrix $A$ at any time is given by (2) for some (possibly time-varying) $\lambda \in \Lambda$.

We refer to $\mathcal{D}(x, \mathbb{A},\{0\})$ (i.e. when $\mathbb{W}=\{0\}$ ) as the nominal part of the linear difference inclusion (1).

The main motivation for considering linear difference inclusions of the form (1) lies in the fact that a broad class of systems can be modeled by this form. For example, consider the following uncertain, linear discrete-time system:

$$
\begin{aligned}
& x^{+}=F x+G u+w,(F, G) \in \operatorname{co}(\mathbb{C}), w \in \mathbb{W} \\
& \mathbb{C} \triangleq\left\{\left(F_{i}, G_{i}\right) \in \mathbb{R}^{n \times n} \times \mathbb{R}^{n \times m} \mid i \in \mathbb{N}_{q}^{+}\right\}
\end{aligned}
$$

It is well known that system (4) with $\mathbb{W}=\{0\}$ is stabilized by $u=K x$ if there exists a solution to the following, possibly conservative, linear matrix inequality problem [27]:

$$
\left(F_{i}+G_{i} K\right)^{T} P\left(F_{i}+G_{i} K\right)-P<0, P=P^{T}>0, \forall i \in \mathbb{N}_{q}^{+}
$$

Motivated by (5) with $A_{i} \triangleq F_{i}+G_{i} K$, we assume that:

Assumption 2 There exists a pair $(P, \psi) \in \mathbb{R}^{n \times n} \times(0,1)$ such that $P=P^{T}>0$ and

$$
A_{i}^{T} P A_{i}-P \leq-\psi P, \forall i \in \mathbb{N}_{q}^{+}
$$

Recalling results in [28, Section 3] on stability of linear difference inclusions when $\mathbb{W}=\{0\}$, it follows that if (6) holds then the linear difference inclusion (1) with $\mathbb{W}=\{0\}$ is Absolutely Asymptotically Stable (AAS) [28] so that $\lim _{k \rightarrow \infty} x(k) \rightarrow 0$, where $x(k)$ is generated by $x(i+1)=A(i) x(i), x(0)=x_{0}$ for any $A(i) \in \mathbb{A}$ and any $x_{0} \in \mathbb{R}^{n}$. It is also known that if (6) holds then the nominal part of the linear difference inclusions (1) (with $\mathbb{W}=\{0\}$ ) is AAS for all $A \in \operatorname{co}(\mathbb{A})[27,33]$. We also remark that the recent results reported in [34] can, in principle, be employed to relax Assumption 2, however we do not elaborate here on such a possibility.

Given a non-empty set $X \subset \mathbb{R}^{n}$, we use the following standard notation for the one step forward reachable set:

$$
\mathcal{D}(X, \mathbb{A}, \mathbb{W}) \triangleq\{A x+w \mid x \in X, A \in \operatorname{co}(\mathbb{A}), w \in \mathbb{W}\}
$$

The following two definitions are standard definitions in set invariance theory (see [1, Section 2] and [10, Section 4]):

Definition 4 A set $\Omega$ is a robust positively invariant $(R P I)$ set of the difference inclusion $(1)$ if $\mathcal{D}(\Omega, \mathbb{A}, \mathbb{W}) \subseteq \Omega$.

Definition 5 The set $D_{\infty}^{e}$ is the minimal robust positively invariant (mRPI) set for the difference inclusion (1) over the class of closed RPI sets, if $D_{\infty}^{e}$ is an RPI set and $D_{\infty}^{e}$ is contained in every closed RPI set for the difference inclusion (1). 
Definition 6 The set $D_{\infty}$ is the minimal convex robust positively invariant ( $m$ CRPI) set for the difference inclusion (1) over class of closed RPI sets, if $D_{\infty}$ is a convex RPI set and $D_{\infty}$ is contained in every closed convex RPI set for the difference inclusion (1).

Clearly, from Definitions 5 and 6 it follows that, provided it exists, $D_{\infty}=\operatorname{co}\left(D_{\infty}^{e}\right)$, because it is possible to show that $\operatorname{co}(\Omega)$ is a RPI set for the difference inclusion (1) if $\Omega$ is such a set.

In order to discuss the convergence of the set sequences (taken in the Hausdorff metric sense) and to clarify our use of the term outer, RPI $\varepsilon$-approximation of the sets we recall the following two definitions:

Definition 7 If $\Omega$ and $\Phi$ are two non-empty, compact sets in $\mathbb{R}^{n}$, then the Hausdorff metric is defined as

$$
\mathcal{H}(\Omega, \Phi) \triangleq \max \left\{\sup _{\omega \in \Phi} d(\omega, \Omega), \sup _{\phi \in \Omega} d(\phi, \Phi)\right\}
$$

where $d(z, \mathcal{Z}) \triangleq \inf _{y \in \mathcal{Z}}\|z-y\|_{p}$

Definition 8 Given a scalar $\varepsilon>0$ and a non-empty set $\Omega \subset \mathbb{R}^{n}$, the set $\Phi \subset \mathbb{R}^{n}$ is an outer $\varepsilon$-approximation of $\Omega$ if $\Omega \subseteq \Phi \subseteq \Omega \oplus \mathbb{B}_{p}^{n}(\varepsilon)$ and an inner $\varepsilon$-approximation of $\Omega$ if $\Phi \subseteq \Omega \subseteq \Phi \oplus \mathbb{B}_{p}^{n}(\varepsilon)$.

It is known that a collection of non-empty compact sets in $\mathbb{R}^{n}$, equipped with the Hausdorff Metric, forms a complete metric space [35]. A direct consequence is that every convergent or Cauchy sequence (whose elements belong to this collection) converges to an element of the space.

We also need the following definition in Section 5, where computational issues for the approximation of $D_{\infty}$ are discussed:

Definition 9 The support function $h_{\mathcal{X}}(\cdot)$ of a set $\mathcal{X} \subset \mathbb{R}^{n}$, evaluated at a vector $\eta \in \mathbb{R}^{n}$, is defined by:

$$
h_{\mathcal{X}}(\eta) \triangleq \sup _{x}\left\{\eta^{T} x \mid x \in \mathcal{X}\right\}
$$

Note that if $\mathcal{X}$ is a polytope then the supremum in Definition 9 is in fact maximum; furthermore, evaluation of $h_{\mathcal{X}}(\eta)$ is a linear programming problem.

For sake of convenience and compactness of the presentation in the sequel of this paper, we introduce the following additional notational convention. Let, for any $k \in \mathbb{N}^{+}, \mathbf{i}_{k} \triangleq\left\{i_{k}, i_{k-1}, \ldots, i_{2}, i_{1}\right\}$ denote a sequence of integer variables such that $i_{j} \in \mathbb{N}_{q}^{+}$for e ach $j \in \mathbb{N}_{k}^{+}$and let for notational convenience $\mathbf{i}_{0} \triangleq\{0\}$. We denote the set of all integer sequences $\mathbf{i}_{k}$ by $\mathcal{I}_{k} \triangleq\left\{\mathbf{i}_{k} \mid i_{j} \in \mathbb{N}_{q}^{+}, j \in \mathbb{N}_{k}^{+}\right\}, \forall k \in \mathbb{N}^{+}$and $\mathcal{I}_{0} \triangleq\left\{\mathbf{i}_{0}\right\}$. Given an arbitrary sequence $\mathbf{i}_{k} \in \mathcal{I}_{k}\left(\mathbf{i}_{k}=\left\{i_{k}, i_{k-1}, \ldots, i_{2}, i_{1}\right\}\right)$ and an integer $l$ such that $l \in \mathbb{N}_{k-1}$ we define $\mathbf{j}_{k-l}\left(\mathbf{i}_{k}\right) \triangleq\left\{i_{k}, i_{k-1}, \ldots, i_{l+1}\right\}$ and $\mathbf{j}_{0}\left(\mathbf{i}_{k}\right) \triangleq \mathbf{i}_{0}$ so that $\mathbf{j}_{k-l}\left(\mathbf{i}_{k}\right) \in \mathcal{I}_{k-l}$ and for example, $\mathbf{j}_{k}\left(\mathbf{i}_{k}\right)=\mathbf{i}_{k}, \mathbf{j}_{k-1}\left(\mathbf{i}_{k}\right)=\left\{i_{k}, i_{k-1}, \ldots, i_{2}\right\}, \ldots, \mathbf{j}_{1}\left(\mathbf{i}_{k}\right)=\left\{i_{k}\right\}$. We define the matrices $\mathcal{A}_{\mathbf{i}_{k}} \triangleq A_{i_{k}} \ldots A_{i_{1}} A_{i_{0}}$ for arbitrary sequence $\mathbf{i}_{k} \in \mathcal{I}_{k}$ and $\mathcal{A}_{\mathbf{i}_{0}} \triangleq I$ where $I$ is the identity matrix and $A_{i_{j}} \in \mathbb{A}$; this notational convention applies to matrices $\mathcal{A}_{\mathbf{j}_{k-l}\left(\mathbf{i}_{k}\right)}$ since $\mathbf{j}_{k-l}\left(\mathbf{i}_{k}\right) \in \mathcal{I}_{k-l}$.

\section{The mRPI set $D_{\infty}^{e}$ and the mCRPI set $D_{\infty}$}

In this section we discuss existence and uniqueness of the mRPI set $D_{\infty}^{e}$ for the linear difference inclusion $\mathcal{D}(x, \mathbb{A}, \mathbb{W})$. Define the set sequence $\left\{D_{k}^{e}\right\}$ by:

$$
D_{k+1}^{e} \triangleq \mathcal{D}\left(D_{k}^{e}, \mathbb{A}, \mathbb{W}\right), k \in \mathbb{N}, D_{0}^{e}=\{0\}
$$

which describes the forward reachable tube $[30,36,37]$ starting from the origin for the difference inclusion (1). A fundamental computational problem with respect to the set sequence $\left\{D_{k}^{e}\right\}$ is the fact that the sets $\left\{D_{k}^{e}\right\}$ are not necessarily convex. Given the exact set $D_{k}^{e}$ of reachable states at time $k$, the convexity of $D_{k+1}^{e}$ is not, generally, preserved due to multiplication of the uncertainty in the system transition matrix $A$ and the state uncertainty. This observation, discussed in [26] for the case $x^{+} \in \mathcal{D}(X, \mathbb{A},\{0\})$, is illustrated by a simple example in Section 6. 


\subsection{Existence and Uniqueness of the mRPI set $D_{\infty}^{e}$}

It follows from (1) and (9) that each $D_{k+1}^{e}, k \in \mathbb{N}$, can be expressed as:

$$
D_{k+1}^{e}=\bigcup_{x \in D_{k}^{e}} \mathcal{D}(x, \mathbb{A}, \mathbb{W})=\bigcup_{x \in D_{k}^{e}}\{A x+w \mid(A, w) \in \operatorname{co}(\mathbb{A}) \times \mathbb{W}\}
$$

The exact calculation of the sets $D_{k}^{e}$ by using (10) requires, clearly, uncountably many operations. Furthermore, the set $D_{k+1}^{e}$ is possibly non-convex even if the set $D_{k}^{e}$ is convex. These facts are also observed in [26] for a simpler case. Since $0 \in \operatorname{interior}(\mathbb{W})$ it follows that $0 \in \operatorname{interior}\left(D_{k}^{e}\right)$ for all $k \in \mathbb{N}^{+}$. Now, since $D_{0}^{e}=\{0\} \subseteq \mathbb{W}=D_{1}^{e}$ and $\mathcal{D}(X, \mathbb{A}, \mathbb{W}) \subseteq \mathcal{D}(Y, \mathbb{A}, \mathbb{W})$ when $X \subseteq Y \subseteq \mathbb{R}^{n}$ it follows by induction that:

$$
D_{k}^{e} \subseteq D_{k+1}^{e}, \forall k \in \mathbb{N}
$$

In order to study the limiting behavior of the set sequence $\left\{D_{k}^{e}\right\}$, we introduce the sets $R_{k}^{e}, k \in \mathbb{N}$ defined by:

$$
R_{k}^{e} \triangleq \mathcal{D}\left(R_{k-1}^{e}, \mathbb{A},\{0\}\right), k \in \mathbb{N}^{+}, R_{0}^{e} \triangleq \mathbb{W}
$$

where $\mathcal{D}(X, \mathbb{A},\{0\})$ is defined by (7). The set $R_{k}^{e}$ is the set of states that can be reached at time $k$, by the nominal part $x^{+} \in \mathcal{D}(x, \mathbb{A},\{0\})$ of the difference inclusion (1), starting from an initial state that belongs to the set $\mathbb{W}$.

It is useful to know the structure of $R_{k}^{e}, D_{k}^{e}$ before proceeding to study their limiting behaviour when $k \rightarrow \infty$; for this reason we establish next result:

Proposition 1 Consider the sets $D_{k}^{e}$ and $R_{k}^{e}, k \in \mathbb{N}$, defined by (9) (or (10)) and (12). Then:

$$
D_{k}^{e} \subseteq D_{k+1}^{e} \subseteq D_{k}^{e} \oplus R_{k}^{e}, \forall k \in \mathbb{N}
$$

(ii) If Assumption 1 holds, the sets $D_{k}^{e}$ and $R_{k}^{e}$, are basic star-shaped sets.

Proof: See appendix B1.

By the proof of Proposition 1 it is clear that the above result hold when $\mathbb{W}$ is a basic star-shaped set.

Exploiting Proposition 1, we establish the following properties of the set sequence $\left\{D_{k}^{e}\right\}$ :

Theorem 1 Suppose Assumptions 1 and 2 hold. Then the set sequence $\left\{D_{k}^{e}\right\}$ defined by (9) satisfies :

(i) There exist $\theta \in(0,1)$ and $\mu<\infty$ such that $D_{k}^{e} \subseteq D_{k+1}^{e} \subseteq \mathcal{D}_{k}^{e} \oplus \theta^{k} \mathbb{B}_{p}^{n}(\mu)$ for all $k \in \mathbb{N}$,

(ii) there exists a compact set $D_{\infty}^{e}$ such that $\mathcal{H}\left(D_{\infty}^{e}, D_{k}^{e}\right) \rightarrow 0$ as $k \rightarrow \infty$.

Proof: See appendix B2.

Q.e.D.

Theorem 1 establishes that $\left\{D_{k}^{e}\right\}$ is a Cauchy sequence of compact sets if Assumptions 1 and 2 hold. Since a family of compact sets equipped with the Hausdorff metric is a complete metric space, $D_{\infty}^{e}$ is the unique limit of this Cauchy sequence and satisfies the following equation:

$$
D_{\infty}^{e}=\mathcal{D}\left(D_{\infty}^{e}, \mathbb{A}, \mathbb{W}\right)
$$

Consequently, robust positive invariance and minimality of the set $D_{\infty}^{e}$ in (14) over the class of the closed RPI sets follows, since (i) $D_{k}^{e} \subseteq D_{k+1}^{e} \subseteq D_{\infty}^{e}, \forall k \in \mathbb{N}$, (ii) the sets $D_{k}^{e}$ are not robust positively invariant and (iii) $\mathcal{D}\left(D_{\infty}^{e}, \mathbb{A}, \mathbb{W}\right)=D_{\infty}^{e}$. However, the main drawback is the fact that the set $D_{\infty}^{e}$ does not generally admit an explicit representation. 


\subsection{The minimal convex RPI set $D_{\infty}$}

The fact that the set $D_{\infty}^{e}$ exists and is unique for the class of closed RPI sets for the linear difference inclusion (1), implies directly existence and uniqueness of the minimal convex closed RPI set $D_{\infty}$ for linear difference inclusion (1). However, since the sets $D_{k}^{e}$ are non-convex, the set $D_{\infty}^{e}$ is non-convex in general. Hence, it is generally difficult to use it in any practicable computational scheme. Furthermore, the use of the mCRPI set $D_{\infty}$ is more appropriate for constrained control problems, when the constraints are convex. Since it is difficult to calculate $D_{\infty}$ from $D_{\infty}^{e}$ we resort to an alternative way to compute the set $D_{\infty}$ and proceed to study the properties of the set sequence $\left\{D_{k}\right\}$ defined by:

$$
D_{k+1} \triangleq \operatorname{co}\left(\bigcup_{j \in \mathbb{N}_{q}^{+}} A_{j} D_{k}\right) \oplus \mathbb{W}, k \in \mathbb{N}, D_{0}=\{0\}
$$

It follows from (15) that, for any finite integer $k \in \mathbb{N}^{+}$, the set $D_{k}$ is a $C$ set, since it is the Minkowski addition of a two $C$ sets $\left(0 \in \operatorname{interior}(\mathbb{W})\right.$ it follows that $0 \in \operatorname{interior}\left(D_{k}\right)$ for all $\left.k \in \mathbb{N}^{+}\right)$. Recalling, the set equality [38, Theorem 1.1.2]

$$
\operatorname{co}(\mathcal{X} \oplus \mathcal{Y})=\operatorname{co}(\mathcal{X}) \oplus \operatorname{co}(\mathcal{Y}), \text { for all } \mathcal{X}, \mathcal{Y} \subseteq \mathbb{R}^{n}
$$

the basic properties of Minkowski set addition and the fact that $\mathbb{W}$ is a $C$ set, we have that:

$$
D_{k+1}=\operatorname{co}\left(\left(\bigcup_{j \in \mathbb{N}_{q}^{+}} A_{j} D_{k}\right) \oplus \mathbb{W}\right)=\operatorname{co}\left(\bigcup_{j \in \mathbb{N}_{q}^{+}}\left(A_{j} D_{k} \oplus \mathbb{W}\right)\right), \forall k \in \mathbb{N}^{+}
$$

An alternative description of the set sequence $\left\{D_{k}\right\}$, useful for the analysis in the sequel, is established by the next result.

Proposition 2 Suppose Assumption 1 holds and consider the set sequence $\left\{D_{k}\right\}$ defined by (15) (or (47)). An equivalent form of that sequence is given by:

$$
D_{k+1} \triangleq \operatorname{co}\left(\bigcup_{\mathbf{i}_{k} \in \mathcal{I}_{k}} C_{\mathbf{i}_{k}}\right)
$$

where the sets $C_{\mathbf{i}_{k}}, \mathbf{i}_{k} \in \mathcal{I}_{k}$, are defined by:

$$
C_{\mathbf{i}_{k}} \triangleq \bigoplus_{l=0}^{k} \mathcal{A}_{\mathbf{j}_{k-l}\left(\mathbf{i}_{k}\right)} \mathbb{W}
$$

or equivalently:

$$
C_{\mathbf{i}_{k}}=\mathcal{A}_{\mathbf{j}_{k}\left(\mathbf{i}_{k}\right)} \mathbb{W} \oplus \mathcal{A}_{\mathbf{j}_{k-1}\left(\mathbf{i}_{k}\right)} \mathbb{W} \oplus \ldots \oplus \mathcal{A}_{\mathbf{j}_{1}\left(\mathbf{i}_{k}\right)} \mathbb{W} \oplus \mathcal{A}_{\mathbf{j}_{0}\left(\mathbf{i}_{k}\right)} \mathbb{W}
$$

Proof: See appendix B3. $\left\{D_{k}^{e}\right\}$ :

Our next step is to exploit Proposition 2 in order to establish a relationship between the set sequences $\left\{D_{k}\right\}$ and

Proposition 3 Suppose Assumption 1 holds and consider the set sequences $\left\{D_{k}^{e}\right\}$ and $\left\{D_{k}\right\}$ defined by (9) (or (10)) and (15). Then

$$
D_{k}=\operatorname{co}\left(D_{k}^{e}\right), \forall k \in \mathbb{N} \text {. }
$$


Providing Assumptions 1 and 2 hold, by Lemma 2 in Appendix A and by Proposition 3 we have:

$$
D_{\infty}=\operatorname{co}\left(D_{\infty}^{e}\right)
$$

where $D_{\infty}$ is the limit in the Hausdorff Metric of the set sequence $\left\{D_{k}\right\}$ (set equality holds since the limits exist). Since, in this case, the set sequence $\left\{D_{k}\right\}$ is Cauchy, $D_{\infty}$ is its unique limit and consequently satisfies:

$$
D_{\infty}=\operatorname{co}\left(\bigcup_{j \in \mathbb{N}_{q}^{+}} A_{j} D_{\infty}\right) \oplus \mathbb{W}
$$

so that $D_{\infty}$ is a convex RPI set. We have managed to describe $D_{\infty}$ as the limit of a Cauchy set sequence. However, the main problem of obtaining an explicit description of $D_{\infty}$ by means of practicable computation remains. It is difficult to obtain an explicit description of the set $D_{\infty}$ even for the simple case when $q=1$ (so that $\mathbb{A}$ is singleton) and the linear difference inclusion (1) is simply a linear time-invariant system subject to additive, bounded state disturbances and $D_{\infty}=D_{\infty}^{e}$, except maybe in some special cases $[1,10,25]$. It is, however, possible to obtain a family of outer RPI $\epsilon$-approximations of the set $D_{\infty}$ by exploiting ideas in [25] - such a family contains a practicably computable outer RPI $\epsilon$-approximations of the set $D_{\infty}$ as demonstrated in the sequel of this paper.

\section{RPI approximations of the sets $D_{\infty}^{e}$ and $D_{\infty}$}

Motivated by the fact that it is very difficult to obtain a simple computational scheme for constructing the sets $D_{\infty}^{e}$ and $D_{\infty}$, we provide in this section a description of three families of RPI sets. One consists of star-shaped RPI sets. This seems to be the first time that star-shaped RPI sets have been defined and studied. The other families consist of convex RPI sets: one contains convex sets that are outer-bounds for the mRPI set and the other contains the set that is tightest convex approximation of the MCRPI set as well as other convex sets. These families contain members that are practicably computable and consequently of practical relevance for control problems involving linear difference inclusions (1), for instance when considering robust control of linear difference inclusions subject to the state and control constraints. Star-shaped RPI sets are are of practical use when dealing with non-convex state constraints while the other two families are more suitable for the case of the convex constraints.

\subsection{Family of Star-Shaped RPI Sets}

A family of star-shaped RPI sets can be obtained by considering the set sequence $\left\{S_{k}\right\}$ defined by:

$$
S_{k+1} \triangleq \bigoplus_{j=0}^{k} R_{j}^{e}, k \in \mathbb{N}, S_{0} \triangleq\{0\}
$$

where the sets $R_{k}^{e}, k \in \mathbb{N}$, are defined in (12). When $\mathbb{W}$ is a $\mathrm{C}$ or basic star-shaped set with the origin in its interior, the sets $S_{k}$ are star-shaped sets for any finite $k \in \mathbb{N}^{+}$, since they are the Minkowski sum of a finite number of star-shaped sets [29, Chapter 5, Section 5.3]; moreover $0 \in \operatorname{interior}\left(S_{k}\right)$ for all $k \in \mathbb{N}^{+}$, since $0 \in \operatorname{interior}(\mathbb{W})$. The sets $S_{k}$ satisfy:

$$
S_{k} \subseteq S_{k+1}=S_{k} \oplus R_{k}^{e}, \forall k \in \mathbb{N}
$$

The relationship between the set sequences $\left\{D_{k}^{e}\right\}$ and $\left\{S_{k}\right\}$ is established next:

Proposition 4 For the set sequences $\left\{D_{k}^{e}\right\}$ and $\left\{S_{k}\right\}$, defined by (9) and (24):

$$
D_{k}^{e} \subseteq S_{k}, \forall k \in \mathbb{N}
$$


If Assumptions 1 and 2 hold it is possible to demonstrate that $\left\{S_{k}\right\}$ is a Cauchy set sequence, by following the arguments and proof of Theorem 1 ; in which case the limit $S_{\infty}$ of the set sequence $\left\{S_{k}\right\}$ exists, is unique and is given by:

$$
S_{\infty}=\bigoplus_{j=0}^{\infty} R_{j}^{e}
$$

The set $S_{\infty}$ satisfies, by Proposition 4 and Theorem 1 and its analogue in Appendix A,:

$$
D_{\infty}^{e} \subseteq S_{\infty}
$$

and, by elementary properties of the linear difference inclusion (1) given in Appendix A, it follows that:

$$
\begin{aligned}
\mathcal{D}\left(S_{\infty}, \mathbb{A}, \mathbb{W}\right) & =\mathcal{D}\left(\bigoplus_{j=0}^{\infty} R_{j}^{e}, \mathbb{A}, \mathbb{W}\right)=\mathcal{D}\left(\bigoplus_{j=0}^{\infty} R_{j}^{e} \oplus\{0\}, \mathbb{A}, \mathbb{W}\right)=\mathcal{D}\left(\bigoplus_{j=0}^{\infty} R_{j}^{e}, \mathbb{A},\{0\}\right) \oplus \mathcal{D}(\{0\}, \mathbb{A}, \mathbb{W}) \\
& \subseteq \bigoplus_{j=1}^{\infty} R_{j}^{e} \oplus \mathbb{W}=\bigoplus_{j=1}^{\infty} R_{j}^{e} \oplus R_{0}^{e}=\bigoplus_{j=0}^{\infty} R_{j}^{e}=S_{\infty}
\end{aligned}
$$

which is the desired robust positive invariance property of the set $S_{\infty}$.

However, the set $S_{\infty}$ is the Minkowski sum of a countably-infinite number of summands and is therefore of limited practical use. Additionally, it is generally very difficult to obtain a non-conservative estimate of the Hausdorff distance between the sets $S_{\infty}$ and $D_{\infty}^{e}$ (or $D_{\infty}$ ). Nevertheless, the main motivation for considering the set sequence $\left\{S_{k}\right\}$ is the fact that this sequence can be used to obtain practicably computable star-shaped RPI sets as demonstrated next.

We now introduce a condition that permits practicable calculation of RPI sets:

$$
R_{s}^{e} \subseteq \alpha \mathbb{W}
$$

where $(s, \alpha) \in \mathbb{N} \times[0,1)$. A direct consequence of Assumptions 1 and 2 is the fact that $R_{k}^{e} \rightarrow\{0\}$ as $k \rightarrow \infty$. Hence there exist pair $(s, \alpha)$ satisfying $s<\infty$ and $\alpha \in(0,1)$ such that (30) holds. Let:

$$
\mathcal{P}_{S} \triangleq\left\{(s, \alpha) \in \mathbb{N} \times[0,1) \mid R_{s}^{e} \subseteq \alpha \mathbb{W}\right\}
$$

For $(s, \alpha) \in \mathcal{P}_{S}$, we define the following set:

$$
S(s, \alpha) \triangleq(1-\alpha)^{-1} \bigoplus_{j=0}^{s-1} R_{j}^{e}
$$

We establish the robust positive invariance of the sets $S(s, \alpha)$ for arbitrary $(s, \alpha) \in \mathcal{P}_{S}$.

Theorem 2 Suppose that Assumptions 1 and 2 hold. Then, the set $\mathcal{P}_{S}$ defined in (31) is non-empty. Moreover, given any pair $(s, \alpha) \in \mathcal{P}_{S}$ the set $S(s, \alpha)$ of (32) is a star-shaped RPI set for linear difference inclusion (1) and $D_{\infty}^{e} \subseteq S_{\infty} \subseteq S(s, \alpha)$.

Proof: See appendix B6.

Q.e.D.

Theorem 2 provides the description of a family $\mathcal{S}_{S}$ of star-shaped RPI sets defined by:

$$
\mathcal{S}_{S} \triangleq\left\{S(s, \alpha) \mid(s, \alpha) \in \mathcal{P}_{S}\right\}
$$


for which

$$
\mathcal{D}(S(s, \alpha), \mathbb{A}, \mathbb{W}) \subseteq S(s, \alpha), \forall S(s, \alpha) \in \mathcal{S}_{S}
$$

However, the main computational issues (such as, for example, complexity, explicit description and non-convexity) still remain. Since the computation of the sets $R_{k}^{e}$ and hence $S_{k}$, is cumbersome, the results of Theorem 2 are theoretically interesting but of limited use. Motivated by this fact and the fact that most practical control problems are subject to convex state-control constraints we turn our attention to practicably computable convex RPI sets for linear difference inclusion (1).

\subsection{Family of "Outer-Bounding" Convex RPI Sets}

Next we study a family of "outer-bounding" convex RPI sets which have been considered in a conference paper [31]. Consider the set sequence $\left\{F_{k}\right\}$ defined by:

$$
F_{k+1} \triangleq \bigoplus_{j=0}^{k} R_{j}, k \in \mathbb{N}, F_{0} \triangleq\{0\}
$$

where the sets $R_{k}$ are defined by:

$$
R_{k} \triangleq \operatorname{co}\left(\bigcup_{\mathbf{i}_{k} \in \mathcal{I}_{k}} \mathcal{A}_{\mathbf{i}_{k}} \mathbb{W}\right), k \in \mathbb{N}^{+}, R_{0} \triangleq \mathbb{W}
$$

Note that, when $\mathbb{W}$ is a $\mathrm{C}$ set, the sets $F_{k}$ are $\mathrm{C}$ sets for any finite $k \in \mathbb{N}^{+}$, since they are the Minkowski sum of a finite number of $\mathrm{C}$ sets $\left(0 \in \operatorname{interior}\left(F_{k}\right)\right.$ for all $k \in \mathbb{N}^{+}$, since $\left.0 \in \operatorname{interior}(\mathbb{W})\right)$. We first establish the relationship between the set sequences $\left\{R_{k}^{e}\right\}$ and $\left\{R_{k}\right\}$ :

Proposition 5 Suppose Assumption 1 holds and consider the set sequences $\left\{R_{k}^{e}\right\}$ and $\left\{R_{k}\right\}$ defined by (12) and (36) respectively. Then

$$
R_{k}=\operatorname{co}\left(R_{k}^{e}\right), \forall k \in \mathbb{N} .
$$

Proof: See appendix B\%.

By exploiting the results of Lemma 2 and Proposition 5, we can establish the following result:

Proposition 6 Suppose Assumption 1 holds and consider the set sequences $\left\{S_{k}\right\}$ and $\left\{F_{k}\right\}$ defined by (24) and (35) respectively. Then

$$
F_{k}=\operatorname{co}\left(S_{k}\right), \forall k \in \mathbb{N} .
$$

Proof: See appendix B8.

If Assumptions 1 and 2 hold, by Lemma 2 and Proposition 6 we have (because the limits exist):

$$
F_{\infty}=\operatorname{co}\left(S_{\infty}\right)
$$

In this case (when Assumptions 1 and 2 hold), from Propositions 4 and 6 it follows that

$$
D_{k}^{e} \subseteq S_{k} \subseteq F_{k}
$$

for any $k \in \mathbb{N}$ as well as for the limit when $k \rightarrow \infty$, since the limits exist,:

$$
D_{\infty}^{e} \subseteq S_{\infty} \subseteq F_{\infty}
$$


When $\mathbb{W}$ is a $C$ set (Assumption 1 ), the condition (30), by Proposition 5 , is equivalent to:

$$
R_{s} \subseteq \alpha \mathbb{W}
$$

where $(s, \alpha) \in \mathbb{N} \times[0,1)$ (because $R_{s} \subseteq \alpha \mathbb{W} \Rightarrow R_{s}^{e} \subseteq \alpha \mathbb{W}$ and $R_{s}^{e} \subseteq \alpha \mathbb{W} \Rightarrow R_{s} \subseteq \alpha \mathbb{W}$ since $\mathbb{W}$ is a $C$ set and $\left.R_{s}=\mathrm{R}_{\mathrm{s}}^{\mathrm{e}}\right)$. We proceed as in the previous subsection and define:

$$
\mathcal{P}_{F} \triangleq\left\{(s, \alpha) \in \mathbb{N} \times[0,1) \mid R_{s} \subseteq \alpha \mathbb{W}\right\}
$$

As in (32), we define

$$
F(s, \alpha) \triangleq(1-\alpha)^{-1} \bigoplus_{j=0}^{s-1} R_{j}, \forall(s, \alpha) \in \mathcal{P}_{F}
$$

and establish the robust positive invariance of the sets $F(s, \alpha)$ for any pair $(s, \alpha) \in \mathcal{P}_{F}$.

Theorem 3 Suppose that Assumptions 1 and 2 hold. Then, the set $\mathcal{P}_{F}$ defined in (43) is non-empty. Moreover, for any pair $(s, \alpha) \in \mathcal{P}_{F}$ the set $F(s, \alpha)$ of (44) is a C RPI set for linear difference inclusion (1) and $D_{\infty}^{e} \subseteq F_{\infty} \subseteq$ $F(s, \alpha)$.

Proof: See appendix B9.

Q.e.D.

As in the previous subsection, Theorem 3 provides a family (set of sets) $\mathcal{S}_{F}$ of $C$ RPI sets defined by:

$$
\mathcal{S}_{F} \triangleq\left\{F(s, \alpha) \mid(s, \alpha) \in \mathcal{P}_{F}\right\}
$$

Any set $F(s, \alpha) \in \mathcal{S}_{F}$ satisfies, by Theorem 3 ,

$$
\mathcal{D}(F(s, \alpha), \mathbb{A}, \mathbb{W}) \subseteq F(s, \alpha)
$$

From the computational point of view, the set sequence $\left\{F_{k}\right\}$ offers advantages since non-convexity is removed. Thus these results could be of practical use. However, it is in general difficult to obtain a non-conservative estimate of the Hausdorff distance between the sets $F_{\infty}$ and $D_{\infty}^{e}$ (or $D_{\infty}$ ) and, hence, between the sets $F(s, \alpha)$ and $D_{\infty}^{e}$ (or $\left.D_{\infty}\right)$. We therefore do not explore further properties of the set sequences $\left\{S_{k}\right\}$ and $\left\{F_{k}\right\}$. Instead we proceed to study in more detail the set sequence $\left\{D_{k}\right\}$ defined by (15). However, we provide an RPI set $F(s, \alpha)$ and $S(s, \alpha)$ for an illustrative example in section 6 .

\subsection{Family of "Minimal" Convex RPI Sets}

It is established in Sub-Section 3.2 that the set sequence $\left\{D_{k}\right\}$ of (6) satisfies $D_{k}=\operatorname{co}\left(D_{k}^{e}\right)$ for all $k \in \mathbb{N}$ as well as $D_{\infty}=\operatorname{co}\left(D_{\infty}^{e}\right)$ if Assumptions 1 and 2 hold. Since, in this case, the set $D_{\infty}$ is the minimal convex RPI set we exploit the properties of the sets $D_{k}$ to obtain a family of convex RPI sets that contains members that can be calculated in finite time. First, a method is established for the calculation of convex RPI sets, based on a simple, but appropriate, scaling of the sets $D_{k}$. Then, an additional condition is given that allows computation of convex RPI sets which are arbitrarily close approximations (using the Hausdorff metric) of $D_{\infty}=\operatorname{co}\left(D_{\infty}^{e}\right)$.

First, we recall that:

$$
D_{k+1} \triangleq \operatorname{co}\left(\bigcup_{j \in \mathbb{N}_{q}^{+}} A_{j} D_{k}\right) \oplus \mathbb{W}, k \in \mathbb{N}, D_{0} \triangleq\{0\}
$$

It is worth mentioning that Proposition 2 implies directly that $D_{k} \subseteq F_{k}$ for any $k \in \mathbb{N}$ and that $D_{\infty} \subseteq F_{\infty}$ (providing Assumptions 1 and 2 hold) - in general $D_{\infty}$ is a strict subset of $F_{\infty}$ (an example in section 6 indicates $D_{\infty} \subset F_{\infty}$ ). 
In order to obtain a characterization of practicably computable convex RPI sets, the following condition is required:

$$
\mathcal{A}_{\mathbf{j}_{s}\left(\mathbf{i}_{s}\right)} \mathbb{W} \subseteq \alpha \mathbb{W}, \forall \mathbf{i}_{s} \in \mathcal{I}_{s}
$$

where, as before, $(s, \alpha) \in \mathbb{N} \times[0,1)$. Since $\mathbb{W}$ is a $C$ set, condition (48) is equivalent to:

$$
\bigcup_{\mathbf{i}_{s} \in \mathcal{I}_{s}} \mathcal{A}_{\mathbf{j}_{s}\left(\mathbf{i}_{s}\right)} \mathbb{W} \subseteq \alpha \mathbb{W}
$$

and consequently to:

$$
\operatorname{co}\left(\bigcup_{\mathbf{i}_{s} \in \mathcal{I}_{s}} \mathcal{A}_{\mathbf{j}_{s}\left(\mathbf{i}_{s}\right)} \mathbb{W}\right) \subseteq \alpha \mathbb{W}
$$

We note that, since $\mathbf{j}_{s}\left(\mathbf{i}_{s}\right)=\mathbf{i}_{s}$ by definition, conditions (42) and (48) are equivalent when $\mathbb{W}$ is convex; equivalence of conditions (30) and (42) is already established so consequently conditions (30), (42) and (48) are all equivalent if Assumption 1 holds.

Let:

$$
\mathcal{P}_{D} \triangleq\left\{(s, \alpha) \in \mathbb{N} \times[0,1) \mid \mathcal{A}_{\mathbf{j}_{s}\left(\mathbf{i}_{s}\right)} \mathbb{W} \subseteq \alpha \mathbb{W}, \forall \mathbf{i}_{s} \in \mathcal{I}_{s}\right\}
$$

Given any pair $(s, \alpha) \in \mathcal{P}_{D}$ we define the following set:

$$
D(s, \alpha) \triangleq(1-\alpha)^{-1} D_{s}
$$

where $D_{s}$ is given by (15) ( or (47)). We now establish the robust positive invariance of the sets $D(s, \alpha)$ :

Theorem 4 Suppose that Assumptions 1 and 2 hold. Then, the set $\mathcal{P}_{D}$ defined in (51) is non-empty. Moreover, for any $(s, \alpha) \in \mathcal{P}_{D}$ the set $D(s, \alpha)$ of $(52)$ is a $C$ RPI set for linear difference inclusion (1) and $D_{\infty}^{e} \subseteq D_{\infty} \subseteq D(s, \alpha)$.

Proof: See appendix B10.

Q.e.D.

Theorem 4 provides a description of the following family $\mathcal{S}_{D}$ of "minimal" C RPI sets:

$$
\mathcal{S}_{D} \triangleq\left\{D(s, \alpha) \mid(s, \alpha) \in \mathcal{P}_{D}\right\}
$$

Theorem 4 can be used to develop and implement an algorithm for the computation of convex RPI approximation of $D_{\infty}$. Clearly, from Theorem 4 , the set $D(s, \alpha)$ is an outer RPI approximation of $D_{\infty}$. However, the former can be a poor approximation of the latter; hence we proceed to present an extension of the results for the LTI systems case, which were reported in [25], in order to provide a way to obtain a set $D(s, \alpha)$ which is an approximation of pre-specified precision to $D_{\infty}$ in that $D_{\infty} \subseteq D(s, \alpha) \subseteq D_{\infty} \oplus \mathbb{B}_{p}^{n}(\varepsilon)$ for an a-priori given $\varepsilon>0$.

\subsubsection{Limiting Behavior of the RPI set $D(s, \alpha)$}

In order to be able to evaluate the quality of the approximation, in the Hausdorff metric sense, we have to study the limiting behaviour of $D(s, \alpha)$ with respect to the increase of $s$ and the decrease of $\alpha$ i.e. how well $D(s, \alpha)$ approximates $D_{\infty}$ if we choose sufficiently large $s$ or a sufficiently small $\alpha$. Given any $\alpha \in[0,1)$, the smallest value of $s$ such that (48) holds is:

$$
s^{0}(\alpha) \triangleq \inf _{s}\left\{s \in \mathbb{N}^{+} \mid R_{s} \subseteq \alpha \mathbb{W}\right\}
$$

The smallest $\alpha$ such that (48) holds for a given $s \in \mathbb{N}^{+}$is:

$$
\alpha^{0}(s) \triangleq \inf _{\alpha}\left\{\alpha \in \mathbb{R}_{+} \mid R_{s} \subseteq \alpha \mathbb{W}\right\}
$$


Note that, for any $\alpha \in(0,1)$ the value of $s^{0}(\alpha)$ in $(54)$ is finite and that $\alpha^{0}(s) \in[0,1)$ if and only if $s$ is sufficiently large.

The following two theorems extend the results established in [25] for linear systems to the class of linear difference inclusions (1).

The first theorem addresses the issue of the limiting behaviour of $D(s, \alpha)$ :

Theorem 5 Suppose Assumptions 1and 2 hold, then

i) $D\left(s, \alpha^{0}(s)\right) \rightarrow D_{\infty}$ as $s \rightarrow \infty$

ii) $D\left(s^{0}(\alpha), \alpha\right) \rightarrow D_{\infty}$ as $\alpha \searrow 0$

Proof: See Appendix B11.

Q.e.D.

Theorem 5 implies that $D(s, \alpha)$ converges to $D_{\infty}$ as $s \rightarrow \infty$ or $\alpha \searrow 0$. Thus, by increasing $s$ and calculating $\alpha$ from (55), or by decreasing $\alpha$ and calculating $s$ from (54), one can obtain a better approximation of $D_{\infty}$. However, given a pre-specified accuracy, it is not clear yet how to obtain a pair $(s, \alpha)$ such that $D(s, \alpha)$ efficiently approximates $D_{\infty}$ with the given accuracy.

This issue is dealt in with the next theorem, which provides conditions on $(s, \alpha)$ which guarantee that $D(s, \alpha)$ is an outer RPI $\varepsilon$-approximation of the mCRPI set $D_{\infty}$.

Theorem 6 Suppose Assumptions 1 and 2 hold, then for all $\varepsilon>0$ there exists a pair $(s, \alpha) \in[0,1) \times \mathbb{N}^{+}$such that (48) and

$$
\alpha(1-\alpha)^{-1} D_{s} \subseteq \mathbb{B}_{p}^{n}(\varepsilon)
$$

hold. Moreover, for any pair $(s, \alpha) \in[0,1) \times \mathbb{N}^{+}$such that (48) and (56) hold, the set $D(s, \alpha)$ is an outer RPI $\varepsilon$-approximation of $D_{\infty}$.

Proof: See Appendix B12.

Q.e.D.

Theorem 6 clearly states that given an a priori $\varepsilon>0$, a collection of $(s, \alpha)$ can be found to satisfy (48) and (56). Then, any set $D(s, \alpha)$ is an outer RPI $\varepsilon$-approximation of $D_{\infty}$, i.e. $D_{\infty} \subseteq D(s, \alpha) \subseteq D_{\infty} \oplus \mathbb{B}_{p}^{n}(\varepsilon)$. Given $\varepsilon>0$, let:

$$
\mathcal{P}_{(D, \varepsilon)} \triangleq\left\{(s, \alpha) \in \mathbb{N} \times[0,1) \mid R_{s} \subseteq \alpha \mathbb{W}, \alpha(1-\alpha)^{-1} D_{s} \subseteq \mathbb{B}_{p}^{n}(\varepsilon)\right\}
$$

Then, by Theorem 6 , the family of sets $\mathcal{S}_{(D, \varepsilon)} \subseteq \mathcal{S}_{D}$ defined by:

$$
\mathcal{S}_{(D, \varepsilon)} \triangleq\left\{D(s, \alpha) \mid(s, \alpha) \in \mathcal{P}_{(D, \varepsilon)}\right\}
$$

is a family of convex outer RPI $\varepsilon$-approximations of $D_{\infty}$.

Let $M(s) \triangleq \sup _{z}\left\{\|z\|_{p} \mid z \in D_{s}\right\}$ and $M_{\infty} \triangleq \sup _{z}\left\{\|z\|_{p} \mid z \in D_{\infty}\right\}$ and note that these values are attained since $D_{s}$ and $D_{\infty}$ are compact sets. Since $D_{s} \subseteq D_{\infty}, \forall s \in \mathbb{N}$ it follows that $M(s) \leq M_{\infty}$ and

$$
\alpha \leq \varepsilon\left(\varepsilon+M_{\infty}\right)^{-1} \leq \varepsilon(\varepsilon+M(s))^{-1}
$$

Hence, an upper bound for $\alpha$ can be obtained by using (59). Note also that (48) gives a lower bound for $\alpha$ such that $D(s, \alpha)$ is a RPI set that contains $D_{\infty}$.

Remark 1 The results of this subsection can easily be extended to the RPI sets $F(s, \alpha)$ and $S(s, \alpha)$ considered above; this extension is straight-forward and is therefore omitted. 


\section{Computational Issues}

We consider in more detail computational issues regarding the computation of an RPI set $D(s, \alpha)$. However, similar computational schemes can be employed for computation of the sets $F(s, \alpha)$. An algorithm for the computation of an RPI set $D(s, \alpha)$ satisfying $D_{\infty} \subseteq D(s, \alpha) \subseteq D_{\infty} \oplus \mathbb{B}_{p}^{n}(\varepsilon)$ for a given $\varepsilon>0$ can be formulated from Theorems 4 and 6 by observing that the lower and upper bounds imposed on $\alpha$ are specified by (48) (or equivalently by either (30) or (42) since $\mathbb{W}$ is a polytope)and (59) respectively. When $\mathbb{W}$ is a polytope, the pair $(s, \alpha)$ and $M(s)$ can be calculated without having to compute explicitly any of the afore-mentioned sets $D_{k}$ and $R_{k}$.

Suppose that $\mathbb{W} \triangleq\left\{w \in \mathbb{R}^{n} \mid f_{j}^{T} w \leq g_{j}, j \in \mathbb{N}_{l}\right\}$, where $l \in \mathbb{N}_{+}$. The fact that $0 \in \operatorname{interior}(\mathbb{W})$ implies that $\left(f_{j}, g_{j}\right) \in \mathbb{R}^{n} \times(0, \infty), \forall j \in \mathbb{N}_{l}$. By definition 9 and by basic properties of the support function, it can be shown that (48) is satisfied if and only if

$$
f_{j}^{T} \mathcal{A}_{\mathbf{i}_{s}} w \leq \alpha g_{j}, \forall w \in \mathbb{W} \Leftrightarrow h_{\mathbb{W}}\left(\mathcal{A}_{\mathbf{i}_{s}}^{T} f_{j}\right) \leq \alpha g_{j}
$$

for all $\mathbf{i}_{s} \in \mathcal{I}_{s}$ and $j \in \mathbb{N}_{l}$. Furthermore,

$$
\begin{aligned}
& h_{\mathbb{W}}\left(\mathcal{A}_{\mathbf{i}_{s}}^{T} f_{j}\right) \leq \alpha g_{j}, \forall \mathbf{i}_{s} \in \mathcal{I}_{s}, \forall j \in \mathbb{N}_{l} \Leftrightarrow \max _{w \in \mathbb{W}} f_{j}^{T} \mathcal{A}_{\mathbf{i}_{s}} w \leq \alpha g_{j}, \forall \mathbf{i}_{s} \in \mathcal{I}_{s}, \forall j \in \mathbb{N}_{l} \\
& \Leftrightarrow \max _{\mathbf{i}_{s} \in \mathcal{I}_{s}} \max _{w \in \mathbb{W}} f_{j}^{T} \mathcal{A}_{\mathbf{i}_{s}} w \leq \alpha g_{j}, \forall j \in \mathbb{N}_{l} \Leftrightarrow \max _{j \in \mathbb{N}_{l}} \frac{\max _{\mathbf{i}_{s} \in \mathcal{I}_{s}} \max _{w \in \mathbb{W}} f_{j}^{T} \mathcal{A}_{\mathbf{i}_{s}} w}{g_{j}} \leq \alpha
\end{aligned}
$$

Then, equation (61) yields the simple observation that, given $s \in \mathbb{N}^{+}$,

$$
\alpha^{o}(s)=\max _{j \in \mathbb{N}_{l}} \frac{\max _{\mathbf{i}_{s} \in \mathcal{I}_{s}} \max _{w \in \mathbb{W}} f_{j}^{T} \mathcal{A}_{\mathbf{i}_{s}} w}{g_{j}}
$$

Equation (62) allows us to calculate $\alpha^{o}(s)$ for a given $s$ without having to explicitly compute the set $R_{s}$. Of course, $(48)$ is satisfied if and only if $\alpha^{o}(s) \in[0,1)$.

The second issue is the calculation of $M(s)$ without having to calculate $D_{s}$. Since $\mathbb{W}$ (and $D_{s}$ ) are polytopes, it is appropriate to use the infinity norm for the calculation of $M(s)$. Then:

$$
M(s)=\max _{z \in D_{s}}\|z\|_{\infty}=\min _{\gamma}\left\{\gamma \mid D_{s} \subseteq \mathbb{B}_{\infty}^{n}(\gamma)\right\}
$$

which is the minimal value of $\gamma$ for which $D_{s} \subseteq \mathbb{B}_{\infty}^{n}(\gamma)$ holds. The corresponding value of $\gamma$, and hence of $M(s)$, can be computed without having to explicitly compute $D_{s}$, as shown next.

Combining (18) and (63) it follows that:

$$
M(s)=\min _{\gamma}\left\{\gamma \mid \operatorname{co}\left(\bigcup_{\mathbf{i}_{s} \in \mathcal{I}_{s}} C_{\mathbf{i}_{s}}\right) \subseteq \mathbb{B}_{\infty}^{n}(\gamma)\right\}
$$

The set inclusion co $\left(\bigcup_{\mathbf{i}_{s} \in \mathcal{I}_{s}} C_{\mathbf{i}_{s}}\right) \subseteq \mathbb{B}_{\infty}^{n}(\gamma)$ is equivalent to:

$$
C_{\mathbf{i}_{s}} \subseteq \mathbb{B}_{\infty}^{n}(\gamma), \forall \mathbf{i}_{s} \in \mathcal{I}_{s}
$$

Following ideas from [25, Section 4], the simpler set inclusions $C_{\mathbf{i}_{s}} \subseteq \mathbb{B}_{\infty}^{n}(\gamma), \mathbf{i}_{s} \in \mathcal{I}_{s}$ are all satisfied if and only if the following inequalities hold:

$$
\max _{\mathbf{i}_{s} \in \mathcal{I}_{s}} \sum_{l=0}^{s} \max _{w \in \mathbb{W}} e_{j}^{T} \mathcal{A}_{\mathbf{j}_{s-l}\left(\mathbf{i}_{s}\right)} w \leq \gamma \text { and } \max _{\mathbf{i}_{s} \in \mathcal{I}_{s}} \sum_{l=0}^{s} \max _{w \in \mathbb{W}}\left(-e_{j}\right)^{T} \mathcal{A}_{\mathbf{j}_{s-l}\left(\mathbf{i}_{s}\right)} w \leq \gamma, \forall j \in \mathbb{N}_{n}^{+}
$$

where $e_{j}$ is the $j^{\text {th }}$ standard basis vector in $\mathbb{R}^{n}$. 
The smallest value for $\gamma$ can be computed by calculating the maximum of the terms $\max _{\mathbf{i}_{s} \in \mathcal{I}_{s}} \sum_{l=0}^{s} \max _{w \in \mathbb{W}} e_{j}^{T} \mathcal{A}_{\mathbf{j}_{s-l}\left(\mathbf{i}_{s}\right)} w$ and $\max _{\mathbf{i}_{s} \in \mathcal{I}_{s}} \sum_{l=0}^{s} \max _{w \in \mathbb{W}}\left(-e_{j}\right)^{T} \mathcal{A}_{\mathbf{j}_{s-l}\left(\mathbf{i}_{s}\right)} w$ for all $j \in \mathbb{N}_{n}^{+}$:

$$
M(s)=\max _{j \in \mathbb{N}_{n}^{+}}\left\{\max _{\mathbf{i}_{s} \in \mathcal{I}_{s}} \sum_{l=0}^{s} \max _{w \in \mathbb{W}} e_{j}^{T} \mathcal{A}_{\mathbf{j}_{s-l}\left(\mathbf{i}_{s}\right)} w, \max _{\mathbf{i}_{s} \in \mathcal{I}_{s}} \sum_{l=0}^{s} \max _{w \in \mathbb{W}}\left(-e_{j}\right)^{T} \mathcal{A}_{\mathbf{j}_{s-l}\left(\mathbf{i}_{s}\right)} w\right\}
$$

The values for $\alpha^{o}(s)$ and $M(s)$ can be computed from (62) and (67). The results of the above analysis can now be used to formulate Algorithm 1 for the calculation of $D(s, \alpha)$.

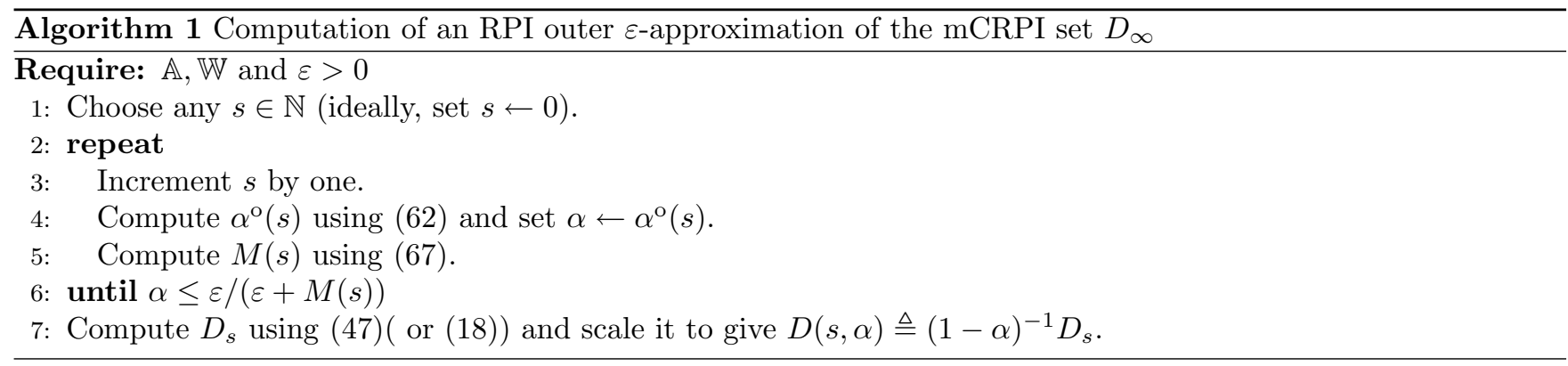

In order to reduce the computational effort for the calculation of $M(s)$, we observe that it is not necessary to calculate directly $\max _{\mathbf{i}_{s} \in \mathcal{I}_{s}} \sum_{l=0}^{s} \max _{w \in \mathbb{W}} e_{j}^{T} \mathcal{A}_{\mathbf{j}_{s-l}\left(\mathbf{i}_{s}\right)} w$ and $\max _{\mathbf{i}_{s} \in \mathcal{I}_{s}} \sum_{l=0}^{s} \max _{w \in \mathbb{W}}\left(-e_{j}\right)^{T} \mathcal{A}_{\mathbf{j}_{s-l}\left(\mathbf{i}_{s}\right)} w$ at each iteration of Algorithm 1. Parts of these sums would have been calculated at a previous iteration; thus it is necessary to compute only $\max _{\mathbf{i}_{s} \in \mathcal{I}_{s}} \max _{w \in \mathbb{W}} e_{j}^{T} \mathcal{A}_{\mathbf{j}_{s}\left(\mathbf{i}_{s}\right)} w$ and $\max _{\mathbf{i}_{s} \in \mathcal{I}_{s}} \max _{w \in \mathbb{W}}\left(-e_{j}\right)^{T} \mathcal{A}_{\mathbf{j}_{s}\left(\mathbf{i}_{s}\right)} w$ and combine this appropriately with already stored results in order to evaluate $M(s)$ in $(67)$ - this requires simple algebraic manipulations and it reduces the computational effort.

Algorithm 1 initially sets $s$ to a fixed value (usually 0 ) and increases it at each step. The values of $\alpha$ and $M(s)$ are calculated in each iteration using (62) and (67). The algorithm stops when the inequality (59) is satisfied, in which case the a-priori specified accuracy $\varepsilon>0$ has been obtained. The $\varepsilon$-approximation $D(s, \alpha)$ of $D_{\infty}$ can then be computed using (47)( or (18)) and simple scaling.

The complexity of Algorithm 1 may increase as the state dimension and $q$ increases. However, the algorithm involves the solution of a number of linear programming problems ((62) and (67)) that can be solved more efficiently than working with set calculations. It is also very useful to note that if $\mathbb{W}=\left\{E w \mid\|w\|_{\infty} \leq 1\right\}$, where $E$ is non-singular, then one can compute $\alpha^{\circ}(s)$ and $M(s)$ without having to resort to solving linear programs, since $\max _{w \in \mathbb{W}} e_{j}^{T} \mathcal{A}_{\mathbf{i}_{k}} w=\left\|E^{T} \mathcal{A}_{\mathbf{i}_{k}}^{T} e_{j}\right\|_{1}$.

\section{$6 \quad$ Illustrative Examples}

To illustrate our results and interesting phenomena occurring when dealing with the reachability analysis of the linear difference inclusions, we provide three simple and constructive $2-D$ examples.

\subsection{Example 1}

Consider an uncertain discrete-time system that takes the form of (4) with:

$$
F_{1}=\left[\begin{array}{cc}
1.2 & 1 \\
0 & 1
\end{array}\right], F_{2}=\left[\begin{array}{cc}
0.8 & 1 \\
0 & 1
\end{array}\right], G_{1}=G_{2}=\left[\begin{array}{l}
1 \\
1
\end{array}\right]
$$

The additive disturbance set is:

$$
\mathbb{W} \triangleq\left\{w \in \mathbb{R}^{2} \mid\|w\|_{\infty} \leq 10\right\}
$$


The nominal part of the uncertain system (68) can be quadratically stabilized by the state feedback controller:

$$
K=\left[\begin{array}{ll}
-1.2 & -1
\end{array}\right]
$$

Assumption 2 is satisfied with

$$
P=\left[\begin{array}{cc}
2.9048 & 0 \\
0 & 1
\end{array}\right], \psi=0.33
$$

The closed loop dynamics are:

$$
A_{1}=\left[\begin{array}{cc}
0 & 0 \\
-1.2 & 0
\end{array}\right], A_{2}=\left[\begin{array}{ll}
-0.4 & 0 \\
-1.2 & 0
\end{array}\right]
$$

or equivalently:

$$
A(a)=\left[\begin{array}{cc}
a & 0 \\
-1.2 & 0
\end{array}\right] \text { and } a \in \mathbf{a} \triangleq\{a \mid-0.4 \leq a \leq 0\}
$$

In order to provide a simple example of a star-shaped RPI set $F(s, \alpha)$, we need to characterize the set sequence $\left\{R_{k}^{e}\right\}$. We have that $R_{0}^{e}=\mathbb{W}$ by definition. The exact explicit characterization of the set $R_{1}^{e}$ is obtained as follows:

$$
x \in R_{1}^{e} \Leftrightarrow x=A(a) w,(a, w) \in \mathbf{a} \times \mathbb{W}
$$

It follows that:

$$
x_{1}=a w_{1} \text { and } x_{2}=-1.2 w_{1}
$$

where $x_{i}$ is the $i^{\text {th }}$ coordinate of a vector $x$. From last equation we obtain:

$$
w_{1}=-x_{2} / 1.2 \text { and } a=-1.2 x_{1} / x_{2}
$$

From the bounds on $w_{1}$ and $a$ we obtain:

$$
-10 \leq-x_{2} / 1.2 \leq 10 \text { and }-0.4 \leq-1.2 x_{1} / x_{2} \leq 0
$$

Solving this set of inequalities we find that:

$$
\begin{aligned}
R_{1}^{e} & =R_{11}^{e} \bigcup R_{12}^{e} \\
R_{11}^{e} & \triangleq\left\{x \in \mathbb{R}^{2} \mid 0 \leq x_{2} \leq 12,3 x_{1}-x_{2} \leq 0,-x_{1} \leq 0\right\} \\
R_{12}^{e} & \triangleq\left\{x \in \mathbb{R}^{2} \mid-12 \leq x_{2} \leq 0,-3 x_{1}+x_{2} \leq 0, x_{1} \leq 0\right\}
\end{aligned}
$$

and by (24) $S_{2}=R_{1}^{e} \oplus R_{0}^{e}$. For this particular example it happens to be that $D_{2}^{e}=S_{2}$ where $D_{2}^{e}$ is given by (9) ( (10)). The sets $R_{1}^{e}$ and $R_{1}=\mathrm{co}\left(R_{1}^{e}\right)$ are shown in Figure 1(a), while the sets $D_{2}^{e}=S_{2}$ are shown together with sets $F_{2}=D_{2}=\operatorname{co}\left(D_{2}^{e}\right)=\operatorname{co}\left(S_{2}\right)$ in Figure $1(\mathrm{~b})$. Following the same procedure, with necessary changes, it is easy to verify that:

$$
\begin{aligned}
& R_{2}^{e}=R_{21}^{e} \bigcup R_{22}^{e} \\
& R_{21}^{e} \triangleq\left\{x \in \mathbb{R}^{2} \mid 0 \leq x_{2} \leq 4.8,3 x_{1}-x_{2} \leq 0,-x_{1} \leq 0\right\} \\
& R_{22}^{e} \triangleq\left\{x \in \mathbb{R}^{2} \mid-4.8 \leq x_{2} \leq 0,-3 x_{1}+x_{2} \leq 0, x_{1} \leq 0\right\}
\end{aligned}
$$

and consequently the set $S_{3}$ is then given by $S_{3}=R_{2}^{e} \oplus R_{1}^{e} \oplus R_{0}^{e}$. Unfortunately, the set equality $D_{3}^{e}=S_{3}$ is not true anymore; only set inclusion $D_{3}^{e} \subset S_{3}$ holds. To illustrate this fact we show the sets $S_{3}$ and $F_{3}=\operatorname{co}\left(S_{3}\right)$ in Figure 2 and a vector $p=\left[\begin{array}{lll}-11.6 & 17.2\end{array}\right]^{T}$ that satisfies $p \in S_{3}$. To verify that $p \in S_{3}$ we note that we have (for example):

$$
p=\left[\begin{array}{l}
-1.6 \\
-4.8
\end{array}\right]+\left[\begin{array}{c}
0 \\
12
\end{array}\right]+\left[\begin{array}{c}
-10 \\
10
\end{array}\right] \text { and }\left[\begin{array}{l}
-1.6 \\
-4.8
\end{array}\right] \in R_{2}^{e},\left[\begin{array}{c}
0 \\
12
\end{array}\right] \in R_{1}^{e},\left[\begin{array}{c}
-10 \\
10
\end{array}\right] \in R_{0}^{e} .
$$




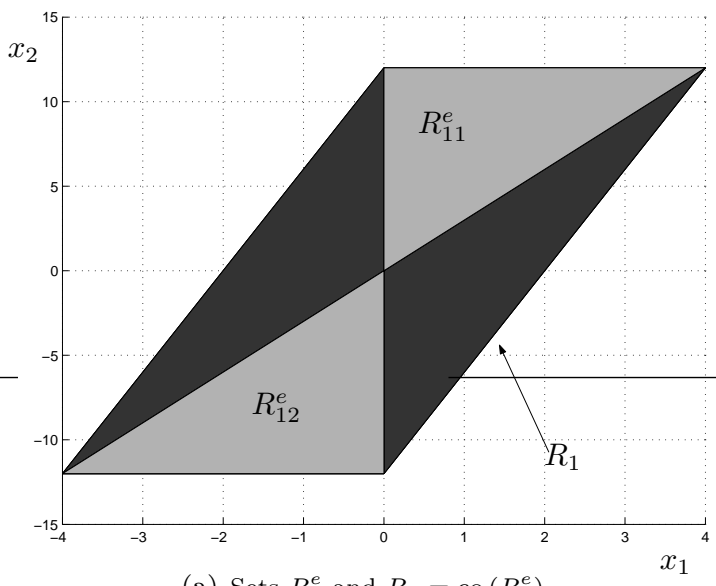

(a) Sets $R_{1}^{e}$ and $R_{1}=\operatorname{co}\left(R_{1}^{e}\right)$.

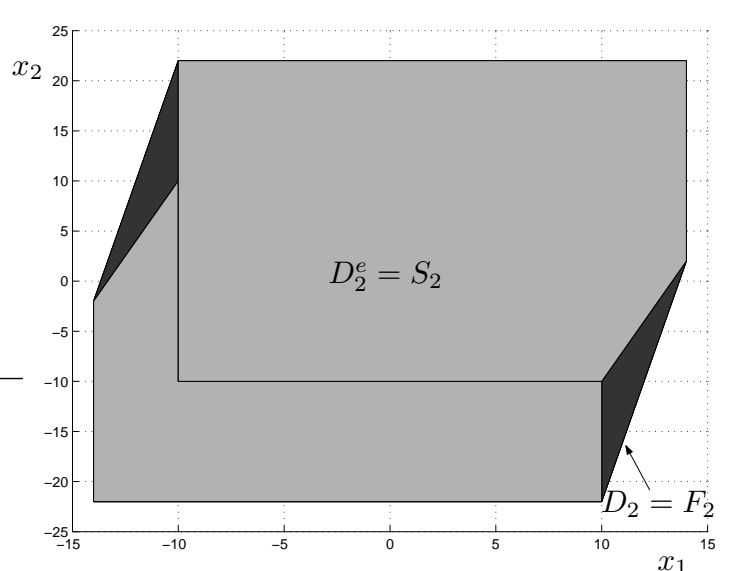

(b) Sets $D_{2}^{e}=S_{2}$ and $F_{2}=D_{2}=\operatorname{co}\left(D_{2}^{e}\right)=\operatorname{co}\left(S_{2}\right)$.

Figure 1: Sets $R_{1}^{e}, R_{1}, D_{2}=F_{2}$ and $D_{2}^{e}=S_{2}$

To show that $D_{3}^{e}$ is not equal to $S_{3}$ (but only $D_{3}^{e} \subset S_{3}$ ) we show that there exists no uncertainty realization resulting in vector $p$ by contradiction. Suppose that $p \in D_{3}^{e}$ in which case we would have:

$$
p=A(a(2)) A(a(1)) w(0)+A(a(2)) w(1)+w(2), a(1), a(2) \in \mathbf{a}, w(0), w(1), w(2) \in \mathbb{W}
$$

The last equation is equivalent to:

$$
\begin{aligned}
-11.6 & =a(2) a(1) w_{1}(0)+a(2) w_{1}(1)+w_{1}(2) \\
17.2 & =-1.2 a(1) w_{1}(0)-1.2 w_{1}(1)+w_{2}(2)
\end{aligned}
$$

From (82b) it follows that:

$$
a(1) w_{1}(0)+w_{1}(1)=\frac{w_{2}(2)-17.2}{1.2}
$$

which when substituted in (82a) yields:

$$
-11.6-w_{1}(2)=\frac{a(2)\left(w_{2}(2)-17.2\right)}{1.2}
$$

Solving (84) for $a(2)$ we have:

$$
a(2)=\frac{13.92+1.2 w_{1}(2)}{17.2-w_{2}(2)}
$$

Using bounds on $a(2)$ we further have:

$$
-0.4 \leq \frac{13.92+1.2 w_{1}(2)}{17.2-w_{2}(2)} \leq 0
$$

or equivalently, after some elementary algebraic manipulations:

$$
0 \leq \frac{104+4 w_{1}(2)}{34.4-2 w_{2}(2)} \text { and } \frac{69.6+6 w_{1}(2)}{34.4-2 w_{2}(2)} \leq 0
$$

Since $-10 \leq w_{2}(2) \leq 10$ it follows that $14.4 \leq 34.4-2 w_{2}(2) \leq 54.4$ so that the last inequalities reduce to:

$$
0 \leq 104+4 w_{1}(2) \text { and } 69.6+6 w_{1}(2) \leq 0 \text { or equivalently }-26 \leq w_{1}(2) \leq-11.6
$$




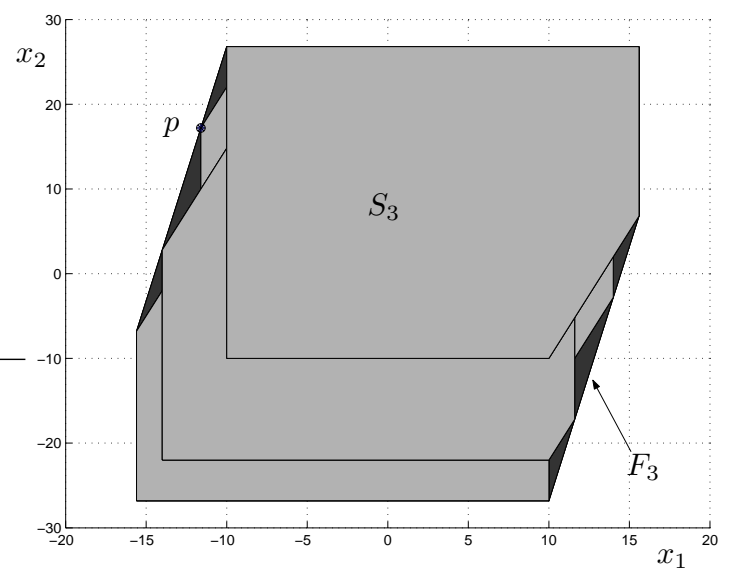

Figure 2: Set $S_{3}$ and vector $p$

which contradicts the bounds on $w_{1}(2)\left(-10 \leq w_{1}(2) \leq 10\right)$; consequently $p \notin D_{3}^{e}$ and $D_{3}^{e}$ is not equal to $S_{3}$. To illustrate a star-shaped RPI set $S(s, \alpha)$, we observe that for instance:

$$
R_{2}^{e} \subseteq 0.48 \mathbb{W}
$$

Thus by choosing $(s, \alpha)=(2,0.48)$ (in fact any $\alpha$ such that $0.48 \leq \alpha<1$ can be used) we construct the following RPI set:

$$
S(2,0.48)=0.52^{-1} S_{2}
$$

The set $S(2,0.48)$ together with $\operatorname{co}(\mathcal{D}(S(2,048), \mathbb{A}, \mathbb{W}))$ is shown in Figure 3 . It is consequently true that $F(2,0.48)$

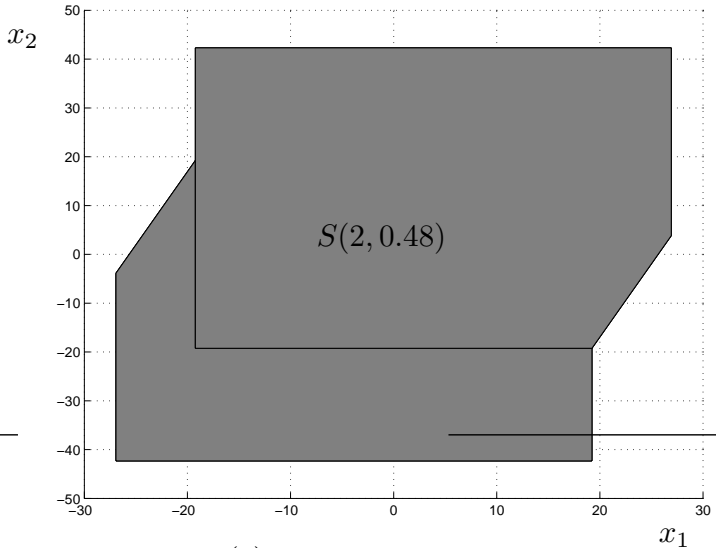

(a) RPI Set $S(2,0.48)$.

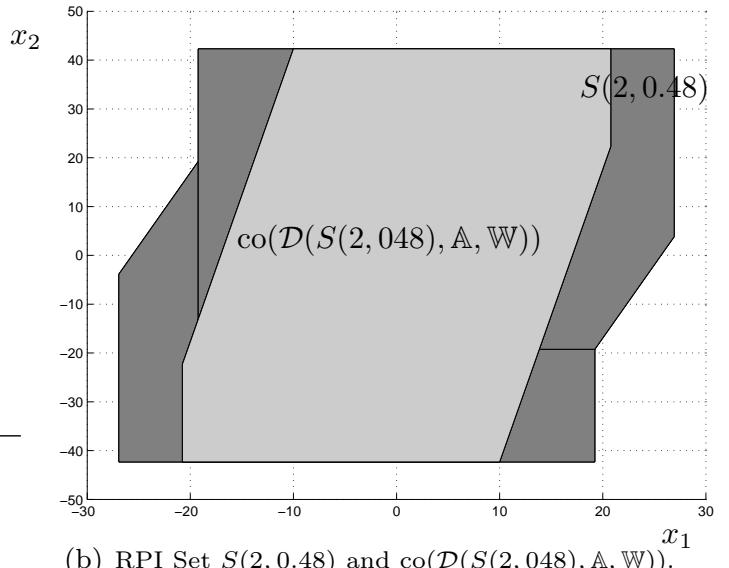

(b) RPI Set $S(2,0.48)$ and $\operatorname{co}(\mathcal{D}(S(2,048), \mathbb{A}, \mathbb{W}))$.

Figure 3: Sets $S(2,0.48)$ and $\operatorname{co}(\mathcal{D}(S(2,048), \mathbb{A}, \mathbb{W}))$

is also an RPI set since $F(2,0.48)=\operatorname{co}(S(2,0.48))$. 
We finally remark that for this particular example:

$$
\begin{aligned}
& R_{k}^{e}=R_{k 1}^{e} \bigcup R_{k 2}^{e} \\
& R_{k 1}^{e} \triangleq\left\{x \in \mathbb{R}^{2} \mid 0 \leq x_{2} \leq 12(0.4)^{k-1}, 3 x_{1}-x_{2} \leq 0,-x_{1} \leq 0\right\} \\
& R_{k 2}^{e} \triangleq\left\{x \in \mathbb{R}^{2} \mid-12(0.4)^{k-1} \leq x_{2} \leq 0,-3 x_{1}+x_{2} \leq 0, x_{1} \leq 0\right\}
\end{aligned}
$$

Consequently, $R_{k}^{e} \rightarrow\{0\}$ exponentially fast (in the Hausdorff Metric) as $k \rightarrow \infty$ as expected by Theorem 1 .

\subsection{Example 2}

Our second example is based on a very simple construction and illustrates that there are degenerate cases for which $D_{\infty}^{e}=D_{\infty}=S_{\infty}=F_{\infty}$ and all of these sets are convex. We observe that for this particular example Assumptions 1 and 2 are violated but despite this fact the sets $D_{\infty}^{e}=D_{\infty}=S_{\infty}=F_{\infty}$ are well defined. Consider the system, similar to the one in sub-section 6.1 :

$$
A(a, b)=\left[\begin{array}{ll}
a & 0 \\
b & 0
\end{array}\right]
$$

where $(a, b)$ is an uncertain pair of bounded scalars (the uncertainty can be arbitrarily large), i.e. $\underline{a} \leq a \leq \bar{a}$ and $\underline{b} \leq b \leq \bar{b}$ where $\underline{a} \leq \bar{a}$ and $\underline{b} \leq \bar{b}$ with scalars $\underline{a}, \bar{a}, \underline{b}$ and $\bar{b}$ being arbitrarily large but bounded. The disturbance set is:

$$
\mathbb{W}=\left\{w \in \mathbb{R}^{2} \mid w_{1}=0, c \leq w_{2} \leq d\right\}, c \leq 0 \leq d
$$

where $(c, d)$ is an arbitrary pair of bounded scalars (arbitrarily large). In this case, the vectors belonging to $\mathbb{W}$ lie in the null-space of $A(a, b)$ for arbitrary $(a, b)$; thus it is trivial to observe that:

$$
D_{\infty}^{e}=D_{\infty}=S_{\infty}=F_{\infty}=\mathbb{W}
$$

This example illustrates that it is, at least in principle, possible to encounter examples for which surprising set equality $D_{\infty}^{e}=D_{\infty}=S_{\infty}=F_{\infty}$ holds and that all of these sets are convex. However, such a construction is possible only for the examples of particular structure; thus this is not a generic case.

\subsection{Example 3}

Our third example illustrates that $D_{k} \subset F_{k}$. Consider the system (4) with

$$
F_{1}=\left[\begin{array}{cc}
1 & 1 \\
1 & 1.1
\end{array}\right], F_{2}=\left[\begin{array}{cc}
1 & 1 \\
1 & 0.9
\end{array}\right], G_{1}=G_{2}=\left[\begin{array}{l}
0 \\
1
\end{array}\right]^{\prime}
$$

and

$$
\mathbb{W} \triangleq\left\{w \in \mathbb{R}^{2} \mid\|w\|_{\infty} \leq 10\right\} .
$$

The feedback controller $K=\left[\begin{array}{ll}-1.4936 & -1.5073\end{array}\right]$ quadratically stabilizes the uncertain system with

$$
P=\left[\begin{array}{cc}
0.07133 & 0.046751 \\
0.046751 & 0.094124
\end{array}\right], \psi=0.22
$$

Figure 4 shows the sets $D_{2}, D_{3}$ and $F_{2}, F_{3}$ and illustrates that $D_{2} \subset F_{2}$ and $D_{3} \subset F_{3}$ indicating that $D_{k} \subset F_{k}$ is a generic case. Figure 5(a) illustrates that approximations $D\left(29,1.4884 \cdot 10^{-7}\right)$ and $F\left(30,5.3359 \cdot 10^{-8}\right)$ of $D_{\infty}$ and $F_{\infty}$, respectively, satisfy $D\left(29,1.4884 \cdot 10^{-7}\right) \subset F\left(30,5.3359 \cdot 10^{-8}\right)$ indicating that $D_{\infty} \subset F_{\infty}$. Both approximations where obtained by setting value $\epsilon=10^{-5}$. Figure $5(\mathrm{~b})$ shows the set sequence $\left\{D_{k}\right\}$ (for $k=1, \ldots, 29$ ) and indicates that the set sequence $\left\{D_{k}\right\}$ is convergent. 


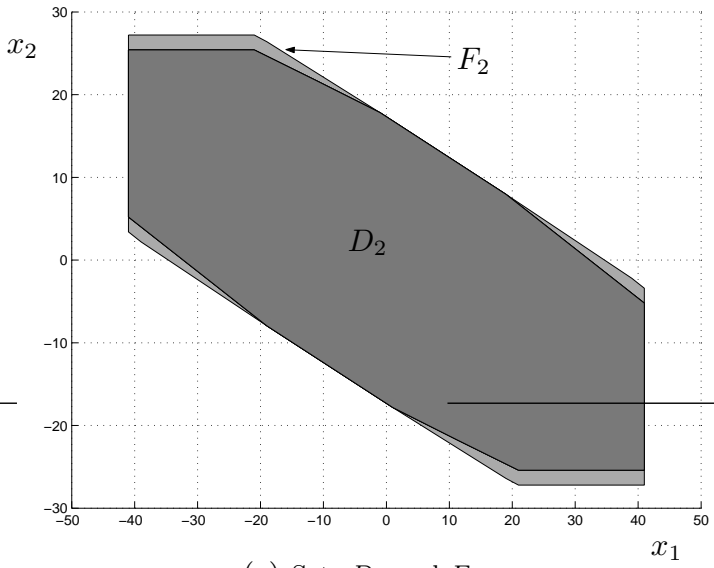

(a) Sets $D_{2}$ and $F_{2}$

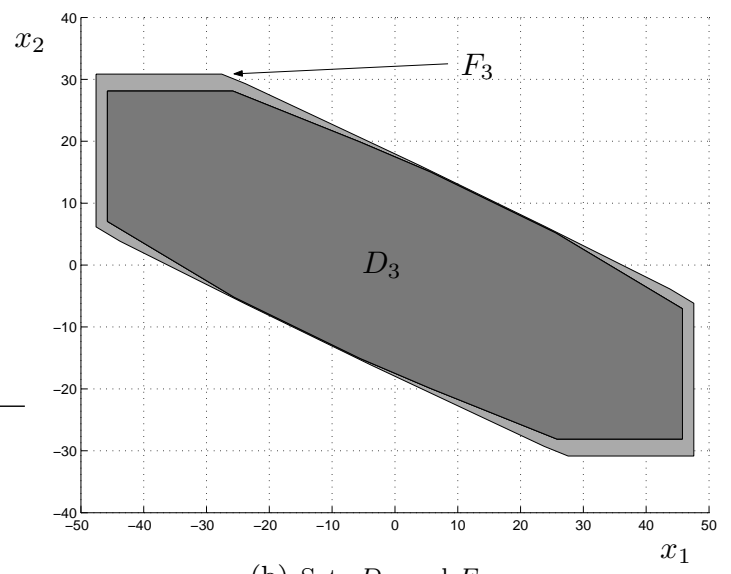

(b) Sets $D_{3}$ and $F_{3}$.

Figure 4: Sets $D_{2}, D_{3}$ and $F_{2}, F_{3}$.

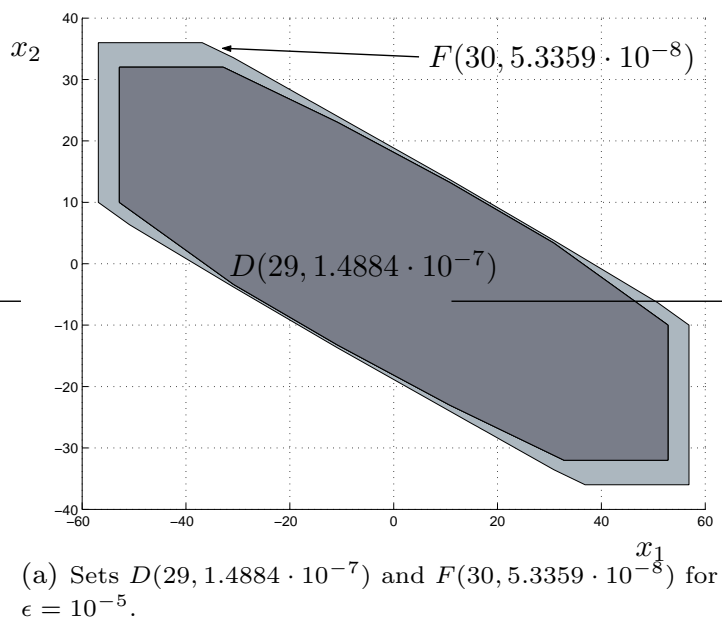

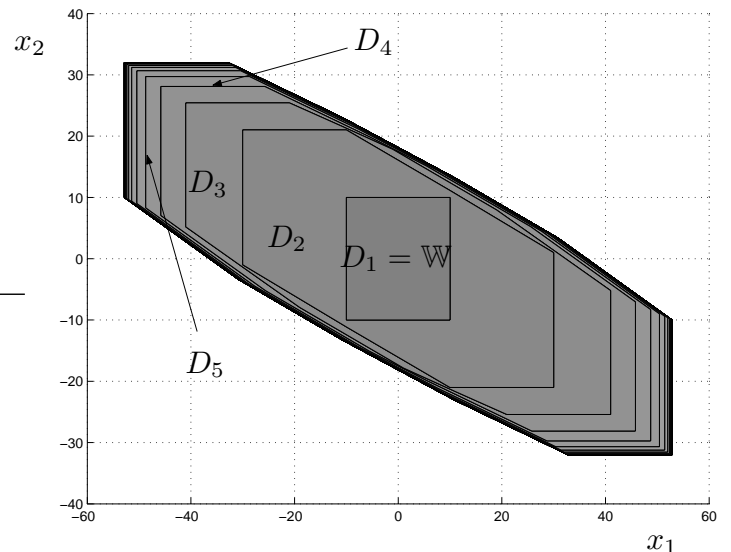

(b) The sets $D_{k}, k=1, \ldots, 29$.

Figure 5: Outer RPI $\epsilon$-approximations and $D_{k}, k=1, \ldots, 29$.

\section{Conclusions}

The novel results reported in this paper further extend the existing research for the computation and approximation of the mCRPI set for autonomous linear discrete-time systems [25]. The results have been extended to address the more general and difficult case of linear difference inclusions. Three families of RPI sets for linear difference inclusion have been characterized. The existence and characterization of basic star-shaped RPI sets for linear difference inclusion is established for the first time. A relevant contribution is a method for the computation of the outer RPI $\varepsilon$-approximation, of the mCRPI set for linear difference inclusions, for an a priori given $\varepsilon>0$. The proposed method is efficient in that it involves the computation of a number of linear programming problems and simple algebraic calculations instead of less tractable calculations with sets. It is in principle possible to further improve computational aspects and this extension is a subject of current research. Several numerical examples are also provided to illustrate interesting phenomena occurring when dealing with robust positive invariance issues for linear difference inclusions. 
The results presented in this paper can be exploited in robust control of linear difference inclusions subject to constraints and additive but bounded disturbances $[5,7,11]$.

\section{APPENDIX A - Necessary technical and preliminary results \\ APPENDIX A1 - Some Properties of Linear Difference Inclusion (1) and its One-Step Forward Reachable Set (7)}

We establish only some elementary properties of $\mathcal{D}(X, \mathbb{A}, \mathbb{W})$, that easily follow from Definition of $\mathcal{D}(X, \mathbb{A}, \mathbb{W})$ and are necessary to simplify our proofs; we also remark that a number of additional properties is easily established.

Lemma 1 Let $X$ and $Y$ be two arbitrary non-empty sets in $\mathbb{R}^{n}$. Then:

(i) Property $\mathrm{X}$ : $\mathcal{D}(X \oplus Y, \mathbb{A}, \mathbb{W}) \subseteq \mathcal{D}(X, \mathbb{A}, \mathbb{W}) \oplus \mathcal{D}(Y, \mathbb{A},\{0\})$,

(ii) Property Y: $\mathcal{D}(\{0\} \oplus Y, \mathbb{A}, \mathbb{W})=\mathcal{D}(\{0\}, \mathbb{A}, \mathbb{W}) \oplus \mathcal{D}(Y, \mathbb{A},\{0\})$,

(iii) Property Z: $\mathcal{D}(X \oplus Y, \mathbb{A},\{0\}) \subseteq \mathcal{D}(X, \mathbb{A},\{0\}) \oplus \mathcal{D}(Y, \mathbb{A},\{0\})$,

Proof:

(i) For arbitrary $z \in \mathcal{D}(X \oplus Y, \mathbb{A}, \mathbb{W})$ holds that $z=A(x+y)+w$ where $(x, y, A, w) \in X \times Y \times \operatorname{co}(\mathbb{A}) \times \mathbb{W}$. We have:

$$
z=A(x+y)+w=A x+A y+w=A x+w+A y
$$

since $z_{1}=A x+w \in \mathcal{D}(X, \mathbb{A}, \mathbb{W})$ and $z_{2}=A y \in \mathcal{D}(Y, \mathbb{A},\{0\})$ it follows that $\mathcal{D}(X \oplus Y, \mathbb{A}, \mathbb{W}) \subseteq \mathcal{D}(X, \mathbb{A}, \mathbb{W}) \oplus$ $\mathcal{D}(Y, \mathbb{A},\{0\})$.

(ii) This property follows from the Definition of $\mathcal{D}(X, \mathbb{A}, \mathbb{W})$.

(iii) This property follows from (i) with $\mathbb{W}=\{0\}$.

Q.e.D.

$W$ e also recall that it is always true that:

(i) $\alpha(X \oplus Y)=\alpha X \oplus \alpha Y$ for any two arbitrary (non-empty) sets $X$ and $Y$ in $\mathbb{R}^{n}$ and an arbitrary $\alpha \in \mathbb{R}$.

(ii) $\operatorname{co}(X \cup Y)=\operatorname{co}(\operatorname{co}(X) \cup \operatorname{co}(Y))$ for any two arbitrary (non-empty) sets $X$ and $Y$ in $\mathbb{R}^{n}$.

\section{APPENDIX A2 - Some Additional Technical Results}

The following result, that can be stated in stronger form, is clear but it is provided here for a sake of completeness.

Lemma 2 Let $\left\{\mathcal{S}_{k} \subset \mathbb{R}^{n}\right\}$ be a Cauchy sequence of compact sets such that $\mathcal{S}_{k} \subseteq \mathcal{S}_{k+1}, \forall k \in \mathbb{N}$. Then:

(i) There exists a compact set $\overline{\mathcal{S}}$ that is a limit of the set sequence $\left\{\mathcal{S}_{k}\right\}$ in the Hausdorff metric sense,

(ii) If $\mathcal{S}_{k}^{c} \triangleq \operatorname{co}\left(\mathcal{S}_{k}\right), \forall k \in \mathbb{N}$, then set sequence $\left\{\mathcal{S}_{k}^{c}\right\}$ is also a Cauchy sequence of compact sets, its limit $\overline{\mathcal{S}}^{c}$ in the Hausdorff metric exists and it satisfies $\overline{\mathcal{S}}^{c}=\operatorname{co}(\overline{\mathcal{S}})$.

Proof:

(i) This claim follows from the facts that: (a) a family of compact sets, each of which is a subset of $\mathbb{R}^{n}$, equipped with Hasudorff metric is a complete metric space [35] and (b) the sequence $\left\{\mathcal{S}_{k}\right\}$ is a Cauchy sequence. Hence, since (i) is true, $\left\{\mathcal{S}_{k}\right\}$ has a limit $\overline{\mathcal{S}}$ in the Hausdorff metric sense that is an element of the space. 
(ii) This claim is a direct consequence of the definition of the set sequence $\left\{\mathcal{S}_{k}^{c}\right\}$.

Q.e.D.

The following result is a direct consequence of Theorem 1 and is provided here for sake of completeness.

Theorem 7 Suppose Assumptions 1 and 2 hold. Then the set sequence $\left\{S_{k}\right\}$ defined by (24) satisfies :

(i) There exist $\theta \in(0,1)$ and $\mu<\infty$ such that $S_{k} \subseteq S_{k+1} \subseteq \mathcal{S}_{k} \oplus \theta^{k} \mathbb{B}_{p}^{n}(\mu)$ for all $k \in \mathbb{N}$,

(ii) there exists a compact set $S_{\infty}$ such that $\mathcal{H}\left(S_{\infty}, S_{k}\right) \rightarrow 0$ as $k \rightarrow \infty$.

Proof: The proof of this result follows the same arguments as the proof of Theorem 1 given in the appendix B2. Q.e.D.

\section{APPENDIX B - Proofs}

\section{APPENDIX B1 - Proof of Proposition 1}

First we show that

$$
D_{k}^{e} \subseteq D_{k+1}^{e} \subseteq D_{k}^{e} \oplus R_{k}^{e}
$$

by principle of mathematical induction. We have $D_{1}^{e}=D_{0}^{e} \oplus R_{0}^{e}$. We assume then, that $D_{k}^{e} \subseteq D_{k+1}^{e} \subseteq D_{k}^{e} \oplus R_{k}^{e}$ and we show that $D_{k+2}^{e} \subseteq D_{k+1}^{e} \oplus R_{k+1}^{e}$. Observe that $\mathcal{D}(X, \operatorname{co}(\mathbb{A}), \mathbb{W}) \subseteq \mathcal{D}(Y, \operatorname{co}(\mathbb{A}), \mathbb{W})$ for any sets $X \subseteq Y \subset \mathbb{R}^{n}$ and recall Property X from Appendix A1. Then

$$
D_{k+2}^{e}=\mathcal{D}\left(D_{k+1}^{e}, \mathbb{A}, \mathbb{W}\right) \subseteq \mathcal{D}\left(D_{k}^{e} \oplus R_{k}^{e}, \mathbb{A}, \mathbb{W}\right) \subseteq \mathcal{D}\left(D_{k}^{e}, \mathbb{A}, \mathbb{W}\right) \oplus \mathcal{D}\left(R_{k}^{e}, \mathbb{A},\{0\}\right)=D_{k+1}^{e} \oplus R_{k+1}^{e}
$$

and the first claim is verified since clearly $D_{k}^{e} \subseteq D_{k+1}^{e}, \forall k \in \mathbb{N}$.

To establish the second claim we only show that $D_{k}^{e}$ is a basic star-shaped set since the same arguments are easily exploited to verify that $R_{k}^{e}$ is a basic star-shaped set. In order to show that $D_{k}^{e}$ is a basic star-shaped set we only have to show, by recalling Definition 3 (with $w_{c}=0$ ), that if $z \in D_{k}^{e}$ then $\lambda z \in D_{k}^{e}$ for any $\lambda \in(0,1]$. Since $D_{k}^{e}$ is a set of the forward reachable tube from the origin of the linear diference inclusion 1 , then for every $z \in D_{k}^{e}$ there exists (at least one feasible combination) $(A(i), w(i)) \in \operatorname{co}(\mathbb{A}) \times \mathbb{W}, i \in \mathbb{N}_{k-1}^{+}$such that

$$
z=A(k-1) A(k-2) \ldots A(2) A(1) w(0)+A(k-1) A(k-2) \ldots A(2) w(1)+\ldots A(k-1) w(k-2)+w(k-1)
$$

and we proceed with the proof assuming that an appropriate selection of this realization is made (for instance minimum-two norm uncertainty realization). Then, for any $\lambda \in(0,1]$

$$
\lambda z=A(k-1) A(k-2) \ldots A(2) A(1) \lambda w(0)+A(k-1) A(k-2) \ldots A(2) \lambda w(1)+\ldots A(k-1) \lambda w(k-2)+\lambda w(k-1)
$$

Since $\lambda \in(0,1]$ and $\mathbb{W}$ is a convex set then $\lambda w(i) \in \mathbb{W}$. If we set $w^{o}(i)=\lambda w(i)$ we have $w^{o}(i) \in \mathbb{W},\left(A(i), w^{o}(i)\right) \in$ $\operatorname{co}(\mathbb{A}) \times \mathbb{W}$ and

$$
\lambda z=A(k-1) A(k-2) \ldots A(2) A(1) w^{o}(0)+A(k-1) A(k-2) \ldots A(2) w^{o}(1)+\ldots A(k-1) w^{o}(k-2)+w^{o}(k-1)
$$

Hence, $\lambda z \in D_{k}^{e}$ and the second claim is verified since $\lambda \in(0,1]$ is arbitrary which completes our proof. 


\section{APPENDIX B2 - Proof of Theorem 1}

This claim is verified as follows:

(i) This fact follows from (13) and Assumptions 1 and 2,

(ii) The part (i) implies that $\mathcal{H}\left(D_{k+1}^{e}, D_{k}^{e}\right) \leq \mu \theta^{k}$ which in turns imply that

$$
\lim _{k \rightarrow \infty} \max _{m \geq 0} \mathcal{H}\left(D_{k+m}^{e}, D_{k}^{e}\right) \leq \theta^{k} \mu(1-\theta)^{-1}
$$

Hence, since $\mu<\infty$ and $\theta \in(0,1)$, it follows that there exists a compact set $D_{\infty}^{e}$ such that $\mathcal{H}\left(D_{\infty}^{e}, D_{k}^{e}\right) \rightarrow 0$ as $k \rightarrow \infty$.

\section{APPENDIX B3 - Proof of Proposition 2}

To establish this claim we resort to the principle of mathematical induction. Suppose that for some $k \in \mathbb{N}$ we have:

$$
D_{k}=\operatorname{co}\left(\bigcup_{\mathbf{i}_{k-1} \in \mathcal{I}_{k-1}} C_{\mathbf{i}_{k-1}}\right)
$$

where the sets $C_{\mathbf{i}_{k-1}}, \mathbf{i}_{k-1} \in \mathcal{I}_{k-1}$ are given by:

$$
C_{\mathbf{i}_{k-1}} \triangleq \bigoplus_{l=0}^{k-1} \mathcal{A}_{\mathbf{j}_{k-1-l}\left(\mathbf{i}_{k-1}\right)} \mathbb{W}
$$

Direct calculation yields:

$$
\begin{aligned}
& D_{k+1}=\operatorname{co}\left(\bigcup_{i \in \mathbb{N}_{q}^{+}} A_{i} D_{k}\right) \oplus \mathbb{W}=\operatorname{co}\left(\bigcup_{i \in \mathbb{N}_{q}^{+}} A_{i} \operatorname{co}\left(\bigcup_{\mathbf{i}_{k-1} \in \mathcal{I}_{k-1}} C_{\mathbf{i}_{k-1}}\right)\right) \oplus \mathbb{W} \\
& =\operatorname{co}\left(\bigcup_{i \in \mathbb{N}_{q}^{+}} \operatorname{co}\left(A_{i} \bigcup_{\mathbf{i}_{k-1} \in \mathcal{I}_{k-1}} C_{\mathbf{i}_{k-1}}\right)\right) \oplus \mathbb{W}=\operatorname{co}\left(\bigcup_{i \in \mathbb{N}_{q}^{+}} \operatorname{co}\left(\bigcup_{\mathbf{i}_{k-1} \in \mathcal{I}_{k-1}} A_{i} C_{\mathbf{i}_{k-1}}\right)\right) \oplus \mathbb{W} \\
& =\operatorname{co}\left(\operatorname{co}\left(\bigcup_{i \in \mathbb{N}_{q}^{+}} \bigcup_{\mathbf{i}_{k-1} \in \mathcal{I}_{k-1}} A_{i} C_{\mathbf{i}_{k-1}}\right)\right) \oplus \mathbb{W}=\operatorname{co}\left(\operatorname{co}\left(\bigcup_{\left(i, \mathbf{i}_{k-1}\right) \in \mathbb{N}_{q}^{+} \times \mathcal{I}_{k-1}} A_{i} C_{\mathbf{i}_{k-1}}\right)\right) \oplus \mathbb{W} \\
& =\operatorname{co}\left(\operatorname{co}\left(\bigcup_{\left(i, \mathbf{i}_{k-1}\right) \in \mathbb{N}_{q}^{+} \times \mathcal{I}_{k-1}} A_{i} C_{\mathbf{i}_{k-1}}\right) \oplus \mathbb{W}\right)=\operatorname{co}\left(\bigcup_{\left(i, \mathbf{i}_{k-1}\right) \in \mathbb{N}_{q}^{+} \times \mathcal{I}_{k-1}} A_{i} C_{\mathbf{i}_{k-1}} \oplus \mathbb{W}\right) \\
& =\operatorname{co}\left(\bigcup_{\left(i, \mathbf{i}_{k-1}\right) \in \mathbb{N}_{q}^{+} \times \mathcal{I}_{k-1}}\left(A_{i} C_{\mathbf{i}_{k-1}} \oplus \mathbb{W}\right)\right)=\operatorname{co}\left(\bigcup_{\mathbf{i}_{k} \in \mathcal{I}_{k}} C_{\mathbf{i}_{k}}\right)
\end{aligned}
$$

The proof is completed by realizing that induction base is trivially true.

\section{APPENDIX B4 - Proof of Proposition 3}

We prove that $D_{k}=\operatorname{co}\left(D_{k}^{e}\right)$ by induction. Hence, we first show that if $D_{k}=\operatorname{co}\left(D_{k}^{e}\right)$ then $D_{k+1}^{e} \subseteq D_{k+1}$, because this means $\operatorname{co}\left(D_{k+1}^{e}\right) \subseteq D_{k+1}$ since $D_{k+1}$ is convex. We subsequently show that $D_{k+1} \subseteq \operatorname{co}\left(D_{k+1}^{e}\right)\left(\right.$ when $\left.D_{k}=\operatorname{co}\left(D_{k}^{e}\right)\right)$ completing the proof. 
Suppose that for some $k \in \mathbb{N}$ we have $\operatorname{co}\left(D_{k}^{e}\right)=D_{k}$. Then for any $y \in D_{k+1}^{e}$ there exist $(x, A, w) \in D_{k}^{e} \times \operatorname{co}(\mathbb{A}) \times \mathbb{W}$ such that

$$
y=A x+w=\left(\sum_{i=1}^{q} \lambda_{i} A_{i}\right) x+w=\sum_{i=1}^{q} \lambda_{i}\left(A_{i} x\right)+w
$$

where $\lambda=\left(\lambda_{1}, \ldots, \lambda_{q}\right) \in \Lambda$ defined in (3) (these are standard convex multipliers). Since $x \in D_{k}^{e} \subseteq \operatorname{co}\left(D_{k}^{e}\right)=D_{k}$ then $A_{i} x \in \operatorname{co}\left(\bigcup_{i \in \mathbb{N}_{q}^{+}} A_{i} D_{k}\right)$ and, furthermore, $\sum_{i=1}^{q} \lambda_{i} A_{i} x \in \operatorname{co}\left(\bigcup_{i \in \mathbb{N}_{q}^{+}} A_{i} D_{k}\right)$. Finally

$$
y=\sum_{i=1}^{q} \lambda_{i}\left(A_{i} x\right)+w \in \operatorname{co}\left(\bigcup_{i \in \mathbb{N}_{q}^{+}} A_{i} D_{k}\right)+\mathbb{W}=D_{k+1}
$$

which implies that $D_{k+1}^{e} \subseteq D_{k+1}$ and the first part of the proof is completed.

We now proceed to prove $D_{k+1} \subseteq \operatorname{co}\left(D_{k+1}^{e}\right)$. Recall from Proposition 2 the alternative definition of $D_{k}$ in equation (18) and the definition of $C_{\mathbf{i}_{k}}$ (19) and (20). It is obvious from the definition of $C_{\mathbf{i}_{k+1}}$ that for any $x \in C_{\mathbf{i}_{k+1}}$ there exists $w(l) \in \mathbb{W}, l \in \mathbb{N}_{k+1}$ such that

$$
x=\sum_{l=0}^{k+1} \mathcal{A}_{\mathbf{j}_{k+1-l}\left(\mathbf{i}_{k+1}\right)} w(l)
$$

which shows that every $x \in C_{\mathbf{i}_{k+1}}$ is a reachable state from the origin in $k+1$ steps. By definition of $D_{k+1}^{e}$, it follows that $x \in D_{k+1}^{e}$ and hence $C_{\mathbf{i}_{k+1}} \subseteq D_{k+1}^{e}$, consequently $\cup_{\mathbf{i}_{k+1} \in \mathcal{I}_{k+1}} C_{\mathbf{i}_{k+1}} \subseteq D_{k+1}^{e}$ and $\operatorname{co}\left(\bigcup_{\mathbf{i}_{k+1} \in \mathcal{I}_{k+1}} C_{\mathbf{i}_{k+1}}\right) \subseteq \operatorname{co}\left(D_{k+1}^{e}\right)$ which yields:

$$
D_{k+1}=\operatorname{co}\left(\bigcup_{\mathbf{i}_{k+1} \in \mathcal{I}_{k+1}} C_{\mathbf{i}_{k+1}}\right) \subseteq \operatorname{co}\left(D_{k+1}^{e}\right)
$$

and the second part of the proof is concluded which completes our proof since clearly induction base is true $D_{0}=D_{0}^{e}$ implies $D_{1}=D_{1}^{e}$ by definition of the set sequences $\left\{D_{k}\right\}$ and $\left\{D_{k}^{e}\right\}$.

\section{APPENDIX B5 - Proof of Proposition 4}

We prove the claim that $D_{k}^{e} \subseteq S_{k}$ for all $k \in \mathbb{N}$ by mathematical induction. Obviously, by definition $D_{0}^{e} \subseteq S_{0}$ and $D_{1}^{e} \subseteq S_{1}$. Then, assume that for some $k \in \mathbb{N}$ we have:

$$
D_{k}^{e} \subseteq S_{k}
$$

Then by Proposition 1,

and consequently, since $D_{k}^{e} \subseteq S_{k}$,

$$
D_{k+1}^{e}=\mathcal{D}\left(D_{k}^{e}, \mathbb{A}, \mathbb{W}\right) \subseteq D_{k}^{e} \oplus R_{k}^{e}
$$

$$
D_{k+1}^{e} \subseteq S_{k} \oplus R_{k}^{e}=S_{k+1}
$$

and the proof is completed.

\section{APPENDIX B6 - Proof of Theorem 2}

The fact that the set $\mathcal{P}_{S}$ defined in (31) is non-empty follows directly from Assumptions 1 and 2 . The claim that the set $S(s, \alpha)$ is a basic star-shaped set is a consequence of the fact that $S_{s}$ is a basic star-shaped set and so is $(1-\alpha)^{-1} S_{s}$. The fact that $D_{\infty}^{e} \subseteq S_{\infty} \subseteq S(s, \alpha)$ follows from Proposition 4 . In order to verify our result it remains to show that $S(s, \alpha)$ is an RPI set for linear difference inclusion for $(s, \alpha) \in \mathcal{P}_{S}$. We have:

$$
\mathcal{D}(S(s, \alpha), \mathbb{A}, \mathbb{W})=\mathcal{D}\left((1-\alpha)^{-1} S_{s}, \mathbb{A}, \mathbb{W}\right)=\mathcal{D}\left((1-\alpha)^{-1} \bigoplus_{j=0}^{s-1} R_{j}^{e}, \mathbb{A}, \mathbb{W}\right)
$$


By Property X established in Appendix A1 we have:

$$
\begin{aligned}
\mathcal{D}\left((1-\alpha)^{-1} \bigoplus_{j=0}^{s-1} R_{j}^{e}, \mathbb{A}, \mathbb{W}\right) & \subseteq \mathcal{D}\left((1-\alpha)^{-1}\{0\}, \mathbb{A}, \mathbb{W}\right) \oplus \bigoplus_{j=0}^{s-1} \mathcal{D}\left((1-\alpha)^{-1} R_{j}^{e}, \mathbb{A},\{0\}\right) \\
& \subseteq \mathbb{W} \oplus \bigoplus_{j=1}^{s}(1-\alpha)^{-1} R_{j}^{e}=\mathbb{W} \oplus(1-\alpha)^{-1} R_{s}^{e} \oplus \bigoplus_{j=1}^{s-1}(1-\alpha)^{-1} R_{j}^{e}
\end{aligned}
$$

However, since $(s, \alpha) \in \mathcal{P}_{S}$ we have that $R_{s}^{e} \subseteq \alpha \mathbb{W}$ and consequently:

$$
\mathbb{W} \oplus(1-\alpha)^{-1} R_{s}^{e} \subseteq \mathbb{W} \oplus(1-\alpha)^{-1} \alpha \mathbb{W}=(1-\alpha)^{-1} \mathbb{W}
$$

Hence,

$$
\mathcal{D}(S(s, \alpha), \mathbb{A}, \mathbb{W}) \subseteq \mathbb{W} \oplus(1-\alpha)^{-1} R_{s}^{e} \oplus \bigoplus_{j=1}^{s-1}(1-\alpha)^{-1} R_{j}^{e} \subseteq(1-\alpha)^{-1} \mathbb{W} \oplus \bigoplus_{j=1}^{s-1}(1-\alpha)^{-1} R_{j}^{e}
$$

and

$$
\mathcal{D}(S(s, \alpha), \mathbb{A}, \mathbb{W}) \subseteq \bigoplus_{j=0}^{s-1}(1-\alpha)^{-1} R_{j}^{e}=S(s, \alpha)
$$

Thus $S(s, \alpha)$ is an RPI set.

\section{APPENDIX B7 - Proof of Proposition 5}

Arguments similar to those employed in the proof of Proposition 3 establish this claim.

\section{APPENDIX B8 - Proof of Proposition 6}

We claim that $F_{k}=\operatorname{co}\left(S_{k}\right)$. This is true because, for any finite $k \in \mathbb{N}$, we have by Proposition 5 and (16):

$$
F_{k}=\bigoplus_{j=0}^{k-1} R_{j}=\bigoplus_{j=0}^{k-1} \operatorname{co}\left(R_{j}^{e}\right)=\operatorname{co}\left(\bigoplus_{j=0}^{k-1} R_{j}^{e}\right)=\operatorname{co}\left(S_{k}\right)
$$

Note that this claim remains valid for the limit $F_{\infty}=\operatorname{co}\left(S_{\infty}\right)$, if Assumptions 1 and 2 hold, due to Theorems 7 and Lemma 2. This completes our proof.

\section{APPENDIX B9 - Proof of Theorem 3}

Observing that conditions (30) and (42) are equivalent (when Assumptions 1 and 2 hold) and that $F_{s}=\operatorname{co}\left(S_{s}\right)$, we conclude that $F(s, \alpha)=\operatorname{co}(S(s, \alpha))$. Moreover, since $S(s, \alpha)$ is an RPI set then, by Theorem $2, F(s, \alpha)=\operatorname{co}(S(s, \alpha))$ is also an RPI set such that $D_{\infty}^{e} \subseteq F_{\infty} \subseteq F(s, \alpha)$.

\section{APPENDIX B10 - Proof of Theorem 4}

The claims that the set $\mathcal{P}_{D}$ (defined in (51)) is non-empty and that $D(s, \alpha),(s, \alpha) \in \mathcal{P}_{D}$ is a $C$ set are direct consequences of Assumptions 1 and 2 and Definition of the set $D(s, \alpha)$. We prove that, given $(s, \alpha) \in \mathcal{P}_{D}$, the set $D(s, \alpha)$ is an RPI set for the linear difference inclusion (1); the claim that $D_{\infty}^{e} \subseteq D_{\infty} \subseteq D(s, \alpha)$ is clearly true by definition of the sets $D_{\infty}^{e}, D_{\infty}$ and $D(s, \alpha)$. Consider a set $D(s, \alpha),(s, \alpha) \in \mathcal{P}_{D}$. We proceed as follows:

$$
\mathcal{D}(D(s, \alpha), \mathbb{A}, \mathbb{W}) \subseteq \operatorname{co}\left(\bigcup_{i \in \mathbb{N}_{q}^{+}} A_{i} D(s, \alpha)\right) \oplus \mathbb{W}
$$


But $D(s, \alpha)$ is defined by:

$$
D(s, \alpha)=(1-\alpha)^{-1} \operatorname{co}\left(\bigcup_{\mathbf{i}_{s-1} \in \mathcal{I}_{s-1}} C_{\mathbf{i}_{s-1}}\right)
$$

where the sets $C_{\mathbf{i}_{s-1}}, \mathbf{i}_{s-1} \in \mathcal{I}_{s-1}$ are given by:

$$
C_{\mathbf{i}_{s-1}} \triangleq \bigoplus_{l=0}^{s-1} \mathcal{A}_{\mathbf{j}_{s-1-l}\left(\mathbf{i}_{s-1}\right)} \mathbb{W}
$$

Following the arguments employed in the proof of Proposition 2, we have

$$
\begin{aligned}
\mathcal{D}(D(s, \alpha), \mathbb{A}, \mathbb{W}) & \subseteq \operatorname{co}\left(\bigcup_{i \in \mathbb{N}_{q}^{+}} A_{i}(1-\alpha)^{-1} \operatorname{co}\left(\bigcup_{\mathbf{i}_{s-1} \in \mathcal{I}_{s-1}} \bigoplus_{l=0}^{s-1} \mathcal{A}_{\mathbf{j}_{s-1-l}\left(\mathbf{i}_{s-1}\right)} \mathbb{W}\right)\right) \oplus \mathbb{W} \\
& =\operatorname{co}\left(\bigcup_{\left(i, \mathbf{i}_{s-1}\right) \in \mathbb{N}_{q}^{+} \times \mathcal{I}_{s-1}}(1-\alpha)^{-1} A_{i} \bigoplus_{l=0}^{s-1} \mathcal{A}_{\mathbf{j}_{s-1-l}\left(\mathbf{i}_{s-1}\right)} \mathbb{W}\right) \oplus \mathbb{W} \\
& =\operatorname{co}\left(\bigcup_{\mathbf{i}_{s} \in \mathcal{I}_{s}}(1-\alpha)^{-1} \bigoplus_{l=0}^{s-1} \mathcal{A}_{\mathbf{j}_{s-l}\left(\mathbf{i}_{s}\right)} \mathbb{W}\right) \oplus \mathbb{W} \\
& =\operatorname{co}\left(\bigcup_{\mathbf{i}_{s} \in \mathcal{I}_{s}}(1-\alpha)^{-1} \bigoplus_{l=0}^{s-1} \mathcal{A}_{\mathbf{j}_{s-l}\left(\mathbf{i}_{s}\right)} \mathbb{W} \oplus \mathbb{W}\right) \\
& =\operatorname{co}\left(\bigcup_{\mathbf{i}_{s} \in \mathcal{I}_{s}}\left((1-\alpha)^{-1} \bigoplus_{l=0}^{s-1} \mathcal{A}_{\mathbf{j}_{s-l}\left(\mathbf{i}_{s}\right)} \mathbb{W} \oplus \mathbb{W}\right)\right) \\
& =\operatorname{co}\left(\bigcup_{\mathbf{i}_{s} \in \mathcal{I}_{s}}\left((1-\alpha)^{-1} \bigoplus_{l=1}^{s-1} \mathcal{A}_{\mathbf{j}_{s-l}\left(\mathbf{i}_{s}\right)} \mathbb{W} \oplus(1-\alpha)^{-1} \mathcal{A}_{\mathbf{j}_{s}\left(\mathbf{i}_{s}\right)} \mathbb{W} \oplus \mathbb{W}\right)\right)
\end{aligned}
$$

Since $(s, \alpha) \in \mathcal{P}_{D}$ we have:

$$
(1-\alpha)^{-1} \mathcal{A}_{\mathbf{j}_{s}\left(\mathbf{i}_{s}\right)} \mathbb{W} \oplus \mathbb{W} \subseteq(1-\alpha)^{-1} \alpha \mathbb{W} \oplus \mathbb{W}=(1-\alpha)^{-1} \mathbb{W}
$$

for all $\mathbf{i}_{s} \in \mathcal{I}_{s}$. Combining the last two equations we obtain:

$$
\begin{aligned}
\mathcal{D}(D(s, \alpha), \mathbb{A}, \mathbb{W}) \subseteq \operatorname{co}\left(\bigcup_{\mathbf{i}_{s} \in \mathcal{I}_{s}}\left((1-\alpha)^{-1} \bigoplus_{l=1}^{s-1} \mathcal{A}_{\mathbf{j}_{s-l}\left(\mathbf{i}_{s}\right)} \mathbb{W} \oplus(1-\alpha)^{-1} \mathbb{W}\right)\right) \\
=\operatorname{co}\left(\bigcup_{\mathbf{i}_{s} \in \mathcal{I}_{s}}(1-\alpha)^{-1} \bigoplus_{l=1}^{s} \mathcal{A}_{\mathbf{j}_{s-l}\left(\mathbf{i}_{s}\right)} \mathbb{W}\right) \\
=\operatorname{co}\left(\bigcup_{\mathbf{i}_{s-1} \in \mathcal{I}_{s-1}}(1-\alpha)^{-1} \bigoplus_{l=0}^{s-1} \mathcal{A}_{\mathbf{j}_{s-1-l}\left(\mathbf{i}_{s-1}\right)} \mathbb{W}\right) \\
=D(s, \alpha)
\end{aligned}
$$

which establishes the RPI property of the set $D(s, \alpha)$ and completes our proof. 


\section{APPENDIX B11 - Proof of Theorem 5}

The proofs of Theorems 5 and 6 follow closely the proofs of Theorem 2 and 3 in [25] and are provided here for sake of completeness.

We first recall that if $\Phi$ is a convex, non-empty, compact set that contains the origin in its interior, for all $\alpha \in[0,1)$ it holds that $\mathcal{H}\left(\Phi,(1-\alpha)^{-1} \Phi\right) \leq \alpha(1-\alpha)^{-1} M$ where $M=\sup _{z \in \Phi}\|z\|_{p}$ is finite [22]. For $s \in \mathbb{N}$, let $M(s) \triangleq \sup _{z \in D_{s}}\|z\|_{p}$.

We proceed to prove (i). We have that $\mathcal{H}\left(D_{s},\left(1-\alpha^{0}(s)\right)^{-1} D_{s}\right) \leq \alpha^{0}(s)\left(1-\alpha^{0}(s)\right)^{-1} M(s)$. Moreover, since $D_{s} \subseteq D_{\infty} \subseteq\left(1-\alpha^{0}(s)\right)^{-1} D_{s}, \mathcal{H}\left(D_{\infty},\left(1-\alpha^{0}(s)\right)^{-1} D_{s}\right) \leq \alpha^{o}(s)\left(1-\alpha^{o}(s)\right)^{-1} M(s)$. Assumption 2 and the fact that $M(s) \leq M_{\infty}, \forall s \in \mathbb{N}$ and $M_{\infty} \triangleq \sup _{z \in D_{\infty}}\|z\|_{p}$ is finite, yields that $\alpha^{0}(s) \rightarrow 0$ as $s \rightarrow \infty$. Thus, $\mathcal{H}\left(D_{\infty},(1-\right.$ $\left.\left.\alpha^{o}(s)\right)^{-1} D_{s}\right) \rightarrow 0$ as $s \rightarrow \infty$ and hence $D\left(s, \alpha^{o}(s)\right)=\left(1-\alpha^{o}(s)\right)^{-1} D_{s} \rightarrow D_{\infty}$ in the Hausdorff metric.

The proof of (ii) follows similar arguments. We have that $\mathcal{H}\left(D_{s^{0}(\alpha)},(1-\alpha)^{-1} D_{s^{0}(\alpha)}\right) \leq \alpha(1-\alpha)^{-1} M\left(s^{0}(\alpha)\right)$ and $\mathcal{H}\left(D_{\infty},(1-\alpha)^{-1} D_{s^{0}(\alpha)}\right) \leq \alpha(1-\alpha)^{-1} M\left(s^{0}(\alpha)\right)$. Note that for $\alpha \searrow 0$ we have $s^{0}(\alpha) \rightarrow \infty$. Hence, $\mathcal{H}\left(D_{\infty},(1-\right.$ $\left.\alpha)^{-1} D_{s^{0}(\alpha)}\right) \rightarrow 0$ as $\alpha \searrow 0$ and therefore $D\left(s^{0}(\alpha), \alpha\right) \rightarrow D_{\infty}$ as $\alpha \searrow 0$.

\section{APPENDIX B12 - Proof of Theorem 6}

We refer to the proof of Theorem 5 for the definition of $M_{\infty}$. Let $\varepsilon>0$ and recall that $0<M_{\infty}<\infty$ and $D_{s} \subseteq D_{\infty}$ for all $s \in \mathbb{N}$. Since $D_{s}$ and $D_{\infty}$ are convex and contain the origin, it follows that $\alpha(1-\alpha)^{-1} D_{s} \subseteq \alpha(1-\alpha)^{-1} D_{\infty}$ for any $s \in \mathbb{N}$ and $\alpha \in[0,1)$. Note that the inclusion $\alpha(1-\alpha)^{-1} D_{\infty} \subseteq \mathbb{B}_{p}^{n}(\varepsilon)$ is true if $\alpha(1-\alpha)^{-1} M_{\infty} \leq \varepsilon$ or, equivalently, if $\alpha \leq \varepsilon\left(\varepsilon+M_{\infty}\right)^{-1}$. Hence, (56) is true for any $s \in \mathbb{N}$ and $\alpha \in[0, \bar{\alpha}]$, where $\bar{\alpha} \triangleq \varepsilon\left(\varepsilon+M_{\infty}\right)^{-1} \in[0,1)$. Clearly, (48) is also true if we choose $\alpha \in(0, \bar{\alpha}]$ and $s=s^{\mathrm{o}}(\alpha)$. This establishes the existence of a suitable pair $(\alpha, s)$ such that (48) and (56) hold simultaneously.

Let $(s, \alpha)$ be such that $(48)$ and $(56)$ are true. Since $D(s, \alpha)=(1-\alpha)^{-1} D_{s}$ is a convex and compact set that contains the origin, $D(s, \alpha)=(1-\alpha)^{-1} D_{s}=\left(1+\alpha(1-\alpha)^{-1}\right) D_{s}=D_{s} \oplus \alpha(1-\alpha)^{-1} D_{s}$. Since $D_{s} \subseteq D_{\infty} \subseteq D(s, \alpha) \subseteq$ $D_{s} \oplus \mathbb{B}_{p}^{n}(\varepsilon) \subseteq D_{\infty} \oplus \mathbb{B}_{p}^{n}(\varepsilon)$, it follows that $D(s, \alpha)$ is an RPI, outer $\varepsilon$-approximation of the mCRPI set $D_{\infty}$.

\section{References}

[1] F. Blanchini, Set invariance in control, Automatica 35 (1999) 1747-1767.

[2] E. G. Gilbert, I. Kolmanovsky, Fast reference governors for systems with state and control constraints and disturbance inputs, International Journal of Robust and Nonlinear Control 9 (15) (1999) 1117-41.

[3] A. Bemporad, M. Morari, Robustness in Identification and Control, no. 245 in Lecture Notes in Control and Information Sciences, Springer-Verlag, 1999, Ch. Robust model predictive control: A survey, pp. 207-226, survey paper.

[4] R. Findeisen, L. Imsland, F. Allgöwer, B. A. Foss, State and output feedback nonlinear model predictive control: An overview, European Journal of Control 9 (2-3) (2003) 190-206, survey paper.

[5] D. Q. Mayne, Control of constrained dynamic systems, European Journal of Control 7 (2001) 87-99, survey paper.

[6] D. P. Bertsekas, I. B. Rhodes, On the minimax reachability of target sets and target tubes, Automatica 7 (1971) 233-247.

[7] F. Blanchini, Minimum-time control for uncertain discrete-time linear systems, in: Proc. 31st IEEE Conference on Decision and Control, Vol. 3, Tuczon AZ, USA, 1992, pp. 2629-34.

[8] D. Q. Mayne, W. R. Schroeder, Robust time-optimal control of constrained linear systems, Automatica 33 (12) (1997) 2103-2118. 
[9] S. Raković, P. Grieder, M. Kvasnica, D. Q. Mayne, M. Morari, Computation of invariant sets for piecewise affine discrete time systems subject to bounded disturbances, in: Proc. 43rd IEEE Conference on Decision and Control, Paradise Island, Bahamas, 2004.

[10] I. Kolmanovsky, E. G. Gilbert, Theory and computation of disturbance invariant sets for discrete time linear systems, Mathematical Problems in Engineering: Theory, Methods and Applications 4 (1998) 317-367.

[11] W. Langson, I. Chryssochoos, S. V. Raković, D. Q. Mayne, Robust model predictive control using tubes, Automatica 40 (2004) 125-133.

[12] D. Q. Mayne, M. Seron, S. V. Raković, Robust model predictive control of constrained linear systems with bounded disturbances, Automatica 41 (2005) 219-224.

[13] S. V. Raković, D. Q. Mayne, A simple tube controller for efficient robust model predictive control of constrained linear discrete time systems subject to bounded disturbances, in: Proceedings of the 16th IFAC World Congress IFAC 2005, Praha, Czech Republic, 2005, Invited Session.

[14] E. De Santis, On positively invariant sets for discrete-time linear systems with disturbance: an applications of maximal disturbance sets, IEEE Trans. Automat. Contr. 39 (1) (1994) 245-249.

[15] F. Blanchini, Ultimate boundedness control for uncertain discrete-time systems via set-induced Lyapunov functions, IEEE Trans. Automatic Control 39 (2) (1994) 428-433.

[16] E. De Santis, On invariant sets for constrained discrete time linear systems with disturbances and parametric uncertainties, Automatica 33 (11) (1997) 2033-2039.

[17] E. C. Kerrigan, Robust constraint satisfaction: Invariant sets and predictive control, Ph.D. thesis, University of Cambridge (2000).

[18] K. Kouramas, Control of linear systems with state and control constraints, Ph.D. thesis, Imperial College London (2003).

[19] E. De Santis, M. D. Di Benedetto, L. Berardi, Computation of maximal safe sets for switching systems, IEEE Trans. Automatic Control 49 (2) (2004) 184-195.

[20] S. Raković, P. Grieder, Approximations and properties of the disturbance response set of pwa systems, Tech. Rep. AUT04-02, Automatic Control Lab, ETHZ, Switzerland, http://control.ethz.ch/ (2004).

[21] K. I. Kouramas, J. C. Allwright, Computation of minimal and maximal disturbance invariant sets for uncertain time-varying discrete-time linear systems, IEE Proc. Control Theory and ApplicationsSubmitted.

[22] S. V. Raković, Robust control of constrained discrete-time systems: Characterization and implementation, Ph.D. thesis, Imperial College London (2005).

[23] J. E. Gayek, A survey of techniques for approximating reachable and controllable set, in: Proc. 30th IEEE Conf. Decision and Control, Brighton,U.K., 1991, pp. 1724-1729.

[24] K. Hirata, Y. Ohta, $\varepsilon$-feasible approximation of the state reachable set for discrete time systems, in: Proc. 42nd IEEE Conference on Decision and Control, Maui HI, USA, 2003, pp. 5520-5525.

[25] S. V. Raković, E. C. Kerrigan, K. I. Kouramas, D. Q. Mayne, Invariant approximations of the minimal robustly positively invariant sets, IEEE Transactions on Automatic Control 50 (3) (2005) 406-410.

[26] B. R. Barmish, J. Sarkaran, The propagation of parametric uncertainty via polytopes, IEEE Trans. Automat. Contr. 24 (1979) 346-349. 
[27] E. F. S. Boyd, L. El Ghaoui, V. Balakrishnan, Linear Matrix Inequalities in System and Control Theory, Vol. 15, SIAM, 1994, of Studies in Applied Mathematics.

[28] L. Gurvits, Stability of discrete linear inclusion, Linear Algebra and its Applications 231 (1995) 47-85.

[29] A. M. Rubinov, Abstract convexity and global optimization, Kluwer Academic Publishers, 2000.

[30] A. B. Kurzhanski, T. F. Filippova, On the Theory of Trajectory Tubes: A Mathematical Formalism for Uncertain Dynamics, Viability and Control, in: Advances in Nonlinear Dynamics and Control: A Report from Russia, A.B. Kurzhanski, ed., ser. PSCT 17, Birkhauser, Boston, Basel, Berlin, 1993.

[31] K. I. Kouramas, S. V. Raković, E. C. Kerrigan, J. C. Allwright, D. Q. Mayne, On the minimal robust positively invariant set for linear difference inclusions, in: Proceedings of the 44th IEEE Conference on Decision and Control and European Control Conference ECC 2005, Sevilla, Spain, 2005.

[32] S. V. Raković, K. I. Kouramas, E. C. Kerrigan, J. C. Allwright, D. Q. Mayne, The minimal robust positively invariant set for linear difference inclusions and its robust positively invariant approximations, AutomaticaSubmitted.

[33] M. C. de Oliveira, J. Bernussou, J. C. Geromel, A new discrete-time robust stability condition, Systems and Control Letters (1999) 261-265.

[34] R. Goebel, T. Hu, A. R. Teel, Dual matrix inequalities in stability and performance analysis of linear differential/difference inclusions, Current Trends in Nonlinear Systems and Control, Birkhauser, 2005, Ch. Control and systems theory.

[35] J. P. Aubin, Applied Abstract Analysis, Pure \& Applied Mathematics, Wiley-Interscience, 1977.

[36] J. P. Aubin, Viability theory, Systems \& Control: Foundations \& Applications, Birkhauser, Boston, Basel, Berlin, 1991.

[37] A. B. Kurzhanski, Dynamic optimization for nonlinear target control synthesis, in: Proc. 6th IFAC Symposium - NOLCOS2004, Stuttgart, Germany, 2004.

[38] R. Schneider, Convex bodies: The Brunn-Minkowski theory, Vol. 44, Cambridge University Press, Cambridge, England, 1993, of Encyclopedia of Mathematics and its Applications. 